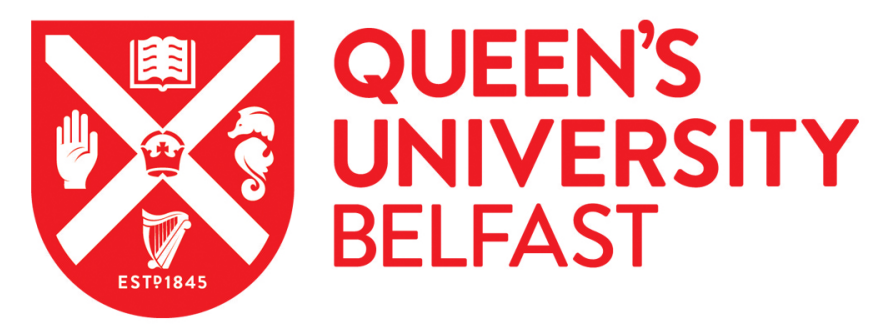

\title{
Domain-wall engineering and topological defects in ferroelectric and ferroelastic materials
}

Nataf, G. F., Guennou, M., Gregg, J. M., Meier, D., Hlinka, J., Salje, E. K. H., \& Kreisel, J. (2020). Domain-wall engineering and topological defects in ferroelectric and ferroelastic materials. Nature Reviews / Physics, 2, 634-648. https://doi.org/10.1038/s42254-020-0235-z

Published in:

Nature Reviews | Physics

Document Version:

Peer reviewed version

Queen's University Belfast - Research Portal:

Link to publication record in Queen's University Belfast Research Portal

Publisher rights

Copyright 2020 Nature Research. This work is made available online in accordance with the publisher's policies. Please refer to any applicable terms of use of the publisher.

\section{General rights}

Copyright for the publications made accessible via the Queen's University Belfast Research Portal is retained by the author(s) and / or other copyright owners and it is a condition of accessing these publications that users recognise and abide by the legal requirements associated with these rights.

Take down policy

The Research Portal is Queen's institutional repository that provides access to Queen's research output. Every effort has been made to ensure that content in the Research Portal does not infringe any person's rights, or applicable UK laws. If you discover content in the Research Portal that you believe breaches copyright or violates any law, please contact openaccess@qub.ac.uk. 


\title{
Domain wall engineering: mastering topological defects in ferroelectric and ferroelastic materials
}

\author{
G. F. Nataf ${ }^{1 *}$, M. Guennou ${ }^{2}$, J. M. Gregg ${ }^{3}$, D. Meier ${ }^{4,5}$, J. Hlinka ${ }^{6}$, E. K. H. Salje ${ }^{7}$ \\ J. Kreisel ${ }^{2}$
}

${ }^{1}$ Department of Materials Science, University of Cambridge, Cambridge, United Kingdom

${ }^{2}$ Department of Physics and Materials Science, University of Luxembourg, Belvaux,

Luxembourg

${ }^{3}$ Centre for Nanostructured Media, School of Mathematics and Physics, Queen's University

Belfast, Belfast, United Kingdom

${ }^{4}$ Department of Materials Science and Engineering, Norwegian University of Science and Technology (NTNU), Trondheim, Norway

${ }^{5}$ Center for Quantum Spintronics, Department of Physics, Norwegian University of Science and Technology (NTNU), Trondheim, Norway

${ }^{6}$ Institute of Physics, Academy of Sciences of the Czech Republic, Praha, Czech Republic

${ }^{7}$ Department of Earth Sciences, University of Cambridge, Cambridge, United Kingdom

*email: gn283@cam.ac.uk

Abstract | Ferroelectric and ferroelastic domain walls are two-dimensional (2D) topological defects with thicknesses approaching the unit cell level. When this spatial confinement is combined with observations of emergent functional properties, such as polarity in non-polar systems or electrical conductivity in otherwise insulating materials, it becomes clear that domain walls represent a new and exciting state of matter. In this review, we discuss the exotic polarisation profiles that can arise at domain walls with multiple order parameters and the different mechanisms that lead to domain wall polarity in non-polar ferroelastic materials. The emergence of energetically degenerate variants of the domain walls themselves suggests the existence of interesting quasi-1D topological defects within such walls. We also provide an overview of the general notions which have been postulated as fundamental mechanisms responsible for domain wall conduction in ferroelectrics. We then discuss the prospect of combining domain walls with transition regions observed at phase boundaries, homo- and heterointerfaces, and other quasi-2D objects, enabling emergent properties beyond those available in today's topological systems.

\section{Key points}

- In ferroelectrics, the emergence of a second polarisation component leads to analogues of Bloch and Néel walls. The stabilization of these walls opens the possibility for quasi-1D topological defects separating wall regions of opposite polarities.

- Polar domain walls in ferroelastics rely on two mechanisms: a polarity imposed by the natural symmetry of strain-compatible domain walls, which can often be described by flexoelectric gradient coupling, and the emergence of a potentially switchable polarity when their natural symmetry is broken.

- Several mechanisms are responsible for domain wall conduction in ferroelectrics: extrinsic intra-bandgap defect states, intrinsic suppression of the conduction band and intrinsic shift of the band structure induced by local electric fields.

- Transition regions occurring at phase boundaries, homo- and heterointerfaces, and other quasi-2D objects probably exist at a smaller length scale, in the vicinity of domain walls, and could lead to exceptional properties and coupling phenomena. 


\section{Introduction}

Ferroic materials (ferromagnets, ferroelectrics, ferroelastics, ferrotoroidics) exhibit a phase transition from a high-symmetry phase, known as the paraphase, to a lowsymmetry phase that exhibits a new physical quantity, characteristic of the symmetry change, called the order parameter (magnetic moment, spontaneous polarisation, spontaneous strain, toroidal moment, respectively) ${ }^{1-3}$. If a material exhibits several ferroic orders, it is referred to as multiferroic ${ }^{1,4,5}$. Due to the energy degeneracy in possible orientations of the order parameter upon cooling from the paraphase, ferroic phases spontaneously exhibit domain structures. Regions of uniform order are called domains and the boundaries between adjacent domains, which are naturally occurring 2D topological defects, are called domain walls. The order parameters can be switched, and thus the domain wall structure engineered, by application of an external field (magnetic field, electric field, stress) ${ }^{1-3}$.

Considering these four groups of materials under the same ferroic umbrella is often useful as they share many analogous behavioural characteristics, such as hysteresis curves, phase transitions and the occurrence of switchable domains. However, there are also important differences when considering their respective domain walls. For example, the contrasting relative magnitudes of dipolar exchange and anisotropy energies cause magnetic domain walls to be relatively broad, typically tens of nanometres; in comparison, ferroelectric and ferroelastic domain walls are extremely narrow, frequently with width at the unit cell level ${ }^{6-8}$. Domain walls in ferroelectrics and ferroelastics can therefore be much more readily considered as genuine $2 \mathrm{D}$ objects.

When dimensional confinement is considered in combination with now widespread observations of polar domain walls in non-polar ferroelastics and dramatically enhanced electrical conductivity in domain walls of ferroelectrics, it becomes clear that ferroelectric and ferroelastic domain walls represent a new 2D topological defect with exciting functionalities. In addition, polarisation variations within domain walls lead to quasi-1D topological defects, which differ from those of established polarisation vortex and skyrmion textures ${ }^{9-12}$. Moreover, since domain walls can be created, eliminated, and moved by an external field, the 2D functionality they offer is not fixed but rather can be actively and dynamically deployed. The potential for mobile conducting ferroelectric domain walls to revolutionise nanoscale electronics and the entire way we think about dynamic circuitry is exciting, representing an intriguing electric alternative to concepts applied in domain-wall-based spintronics devices ${ }^{13,14}$. For memory applications, the possibility to reach very high density of domain walls, up to $1 \%$ of the total volume ${ }^{15-17}$, with their own properties, implies that high information density could be realised, and that wall-related devices could outperform bulk devices by orders of magnitude.

These concepts were envisioned very early-on ${ }^{18}$ and formulated as 'domain wall nanoelectronics ${ }^{19-21}$ or 'domain boundary engineering, ${ }^{\text {,2 }}$, but progress in developing this new technology has been relatively slow. In general, robust, and definitive information about all the fundamental mechanisms responsible for ferroelectric domain wall conduction (BOX 3) are still unclear for many materials, which is one reason why proof-of-principle devices have emerged only recently. These devices include diodes ${ }^{23}$, memories $^{24-29}$ and tunnel junctions ${ }^{30}$. Another approach is to emulate the behaviour of electronic components, such as digital switches and rectifiers, utilising the complex electronic transport phenomena at domain walls ${ }^{31,32}$. Polar domain walls in ferroelastics 
are even further away from applications: thus far, it has merely been shown that the surface potential resulting from the polarisation in the walls can be read and modified with an electron beam ${ }^{33}$ and be used, for example, to tune $2 \mathrm{D}$ electron gases at the nanoscale ${ }^{34}$. In fact, it is likely that memory devices based on polar walls will require first a demonstration of ferroelectricity at the walls, i.e., a switching of the polarisation, before targeting specific application opportunities.

In this review, we discuss the intriguing and non-trivial polarisation configurations that can arise at domain walls and the established mechanisms that can lead to domain wall polarity in non-polar materials. We also provide an overview of the general notions which have been postulated as fundamental mechanisms responsible for domain wall conduction in ferroelectrics. We then suggest that the combination of domain walls with transition regions observed at phase boundaries, homo- and heterointerfaces, and other quasi-2D objects, gives access to novel emergent properties that are yet to be explored.

Our focus is on emergent key functionalities at ferroelectric and ferroelastic domain walls. For a more technical coverage on charged domain walls in these systems, we refer the readers to the review of Bednyakov et al. ${ }^{35}$. We briefly discuss other topological structures in ferroics, which have been reviewed in detail, e.g., by Seidel et $a l{ }^{36,37}$, and we refer the readers to the review of Meier for details on the interplay of ferroelectric and magnetic domain walls in multiferroics ${ }^{38}$.

\section{Domain wall polarisation in polar and non-polar materials}

\section{Phase transitions in ferroelectric domain walls}

Intriguing and non-trivial polarisation configurations beyond the bulk properties can arise at domain walls due to their distinct symmetry. Until recently, the simplest Landau theory of only one order parameter (one-component) was enough to understand the available experimental observations and domain wall properties. Such textbook domain walls are approximated by a hyperbolic tangent $(\tanh )$ profile of the order parameter (see FIG. 1a, 1c and 1d), possibly with small deviations for higher order Landau potentials $^{39,40}$. Local alterations of the properties at the wall have been discussed in this context as early as $1970^{41}$, but no additional symmetry breaking and, in particular, no additional polarisation component, is allowed to emerge at these simple walls. In the special case of ferroelectrics, the standard $180^{\circ}$ domain wall in this model gives rise to a typical polarisation profile, sometimes referred to "Ising wall", whereby the polarisation cancels out at the wall (FIG. 1d).

On the other hand, it was proposed very early on that multiple order parameters, or multi-component order parameters, might open the door to a much richer energy landscape populated by exotic wall profiles leading to polarised - and potentially switchable - domain walls ${ }^{42-44}$. Within this model, a spontaneous polarisation may occur at domain walls, even in an otherwise non-polar material, when the cancellation of the primary order parameter - or instability - at the centre of the domain wall allows a second instability to emerge (FIG. 1b). This domain wall state is bi-stable so that the wall splits spontaneously in two variants ${ }^{42,43}$. In ferroelectrics, the emergence of a second polarisation component leads to Bloch and Néel walls in analogy to ferromagnets (FIG. 1e and 1f). In contrast to magnetic spins, however, the magnitude of the polarisation vector does not have to be constant and both wall profiles may also 
combine polarisation rotations with a reduction of the polarisation, giving rise to complex polarisation profiles. In particular, Bloch- and Néel-type domain walls were heavily researched by various theoretical methods in the $2010 \mathrm{~s}^{45-51}$. The spontaneous symmetry breaking within the wall required for ferroelectric Bloch walls seems to be rare, because polarisation rotation in ferroelectrics comes with substantial costs in elastic energy. Still, it has been theoretically predicted even in standard perovskite ferroelectrics ${ }^{45,49,52-54}$, like barium titanate $\left(\mathrm{BaTiO}_{3}\right)$, lead titanate $\left(\mathrm{PbTiO}_{3}\right)$ and lead zirconate titanate $\left(\mathrm{Pb}\left(\mathrm{Zr}_{\mathrm{x}} \mathrm{Ti}_{1-\mathrm{x}}\right) \mathrm{O}_{3}\right.$, PZT), whereby the special role of flexoelectric coupling has also been investigated ${ }^{50,51}$. These polarisation profiles depend also on the crystallographic orientation of the domain wall normal ${ }^{45,46,53,54}$.

The emergence of energetically degenerate variants of Bloch- and Néel-type walls themselves suggests the existence of interesting quasi-1D topological defects separating wall regions of opposite polarities, which have been discussed as a paradigm for memory devices ${ }^{9,10}$. Other topological defects that recently attracted attention in ferroelectrics are polar skyrmions and bubble domains; however, the latter do not require domain walls (BOX 1$)$.

Even though the basic principles have been known for decades, the relevant length scales are just becoming accessible experimentally so that direct evidence for the theoretical concepts is still scarce. One remarkable recent result by Cherifi-Hertel et al. is the observation of non-Ising components of the domain wall polarisation in lithium tantalate $\left(\mathrm{LiTaO}_{3}\right)$ by optical Second Harmonic Generation $(\mathrm{SHG})^{55}$. Further work along this line is highly desirable in order to understand this signature in detail, and resolve the domain wall structure with atomic scale resolution, as done recently by highresolution transmission electron microscopy (TEM) $)^{56}$, scanning transmission electron microscopy (STEM) $)^{57}$ and $\mathrm{SHG}^{55,57}$ to reveal Néel-like domain walls in PZT.

\section{Ferroelastics and non-polar materials}

While domain walls in ferroelectrics enable unusual polarisation configurations, their impact on the properties in non-polar materials, e.g., ferroelastics and antiferroelectrics, is even more fundamental. Here, domain walls can give rise to an electric polarisation in an otherwise centrosymmetric system. These studies of domain wall polarisations are usually rooted into two complementary approaches: domain wall symmetries and flexoelectricity. One way to describe the symmetries of domain walls is by a so-called layer group, derived systematically using pure geometrical arguments in real space as shown in several works by Janovec ${ }^{58-67}$. The layer group can also be derived by considering symmetries in order parameter space, which allows a natural connection with Landau theory ${ }^{68,69}$. This approach notably yields the remarkable result that all strain-compatible ferroelastic domain walls are non-centrosymmetric and predict possible directions for the polar axis ${ }^{70}$.

Experimentally, very few techniques are in fact able to directly determine the full symmetry of a domain wall as a real 3D object. So far, the point group symmetry has mainly been probed by $\mathrm{SHG}^{71-75}$, performing a polarisation analysis of the incident and emitted light fields. This approach has produced remarkable results, starting with the experimental confirmation of the non-centrosymmetric symmetry of strain-compatible ferroelastic walls ${ }^{71-75}$, but also observations that are at odds with the classical theoretical approaches. In calcium titanate $\left(\mathrm{CaTiO}_{3}\right)$, domain walls were found that have, according to the SHG analysis, a polar axis deviating from the predicted possible 
directions (FIG. 3e $)^{71}$; in lanthanum aluminate $\left(\mathrm{LaAlO}_{3}\right)$, a domain-wall symmetry was proposed $(3 m)$ that does not match the expected layer-group symmetry ${ }^{72}$. The latter case is particularly surprising as $\mathrm{LaAlO}_{3}$ is not expected to exhibit any other instability or structural complexity. Future work will have to clarify these situations.

In addition to their polar point group symmetry, domain walls in ferroelastics come with a gradient of one tensor component of the spontaneous strain tensor. This gradient then creates a polarisation via the flexoelectric effect, which is always symmetryallowed $^{76}$ (FIG 1c). Including this flexoelectric term in the free energy expansion gives a way to model and quantify domain wall polarisation, and many works have used this approach in the context of the recent surging interest in flexoelectric phenomena $^{50,51,76,77}$. Simulations have been reported at the level of a single isolated wall, as well as for larger volumes with the framework of complex ferroelastic patterns, involving several domain walls (FIG. $3 a$ and $3 b)^{76}$. In the latter, it has been shown that junctions between domain walls and kinks in domain walls are key to obtain a macroscopic polarisation, given that, e.g., in the case of simple stripe domains the opposite direction of the polarisation in neighbouring domain walls cancels out and the macroscopic response vanishes ${ }^{76}$.

All this is of course not exclusive to the symmetry breaking mechanisms described earlier (FIG. 1b). In fact, it is now well understood that two mechanisms exist as two possible reasons for the polarity at ferroelastic domain walls: first, a polarity imposed by the symmetry of strain-compatible domain walls ${ }^{70}$, which can be often described by flexoelectric gradient coupling (FIG. 1c) and, second, the accidental and potentially switchable polarity induced by an additional symmetry lowering ${ }^{78}$ (FIG. 1b). Both phenomena can be addressed together by Ginzburg-Landau type modelling ${ }^{69,79,80}$ in single walls or more complex structures. What will have to be clarified is the significance and respective importance of these phenomena with regard to the potential use of a domain wall polarisation as a functional element, i.e., if and how this polarisation can be detected, manipulated, or switched by electric fields.

Recent experimental works on polar domain walls have concentrated on demonstrating the loss of inversion symmetry ${ }^{34,71-75}$, quantifying domain wall polarisation by structural studies ${ }^{81}$, and looking for evidence for characteristic electric or dielectric signatures of this domain wall polarisation ${ }^{11,33,34,82,83}$.

In practice, most experimental studies have been devoted to the perovskites and ferroelastic strontium titanate $\left(\mathrm{SrTiO}_{3}\right)$ and $\mathrm{CaTiO}_{3}$. Both are incipient ferroelectrics with a latent polar instability and are therefore prone to exhibit both possible mechanisms for domain wall polarisation (FIG. $1 \mathrm{~b}$ and 1c). The experimental confirmation that polarity exists in the domain walls of $\mathrm{CaTiO}_{3}$ came by high-resolution TEM conducted by Van Aert et al. ${ }^{81}$, where also the polar distortion amplitude was clarified (FIG. 3c), with off-centre displacements of Ti by 6 picometres at room temperature. The loss of inversion symmetry at the walls was later confirmed by optical SHG microscopy ${ }^{71}$ (FIG. 3e). Domain wall polarisation in $\mathrm{CaTiO}_{3}$ and $\mathrm{SrTiO}_{3}$ was as well invoked as the origin of a significant macroscopic signal in resonance piezoelectric spectroscopy ${ }^{11,82}$ (FIG. 3d), and the sharp contrast observed in low energy electron microscopy (LEEM) ${ }^{33,83}$ (FIG. 3f). In a more original way, domain wall polarisation in $\mathrm{SrTiO}_{3}$ was also shown to change the $\mathrm{LaAlO}_{3} / \mathrm{SrTiO}_{3}$ interface current distribution ${ }^{34}$. 
The investigation of polar domain walls in non-polar materials requires the concerted application of these complementary techniques to understand the complex nanoscale physics involved. TEM is the only way to measure the displacement of the atoms away from the high-symmetry position in order to estimate the amplitude of the polar vector within the domain walls (FIG. 3c) ${ }^{81}$. Resonance piezoelectric spectroscopy measures frequencies of macroscopic mechanical resonances in a sample upon excitation by an AC electric field (FIG. 3d); in the case of materials with non-polar symmetries, these resonances can arise from the motion of polar domain walls ${ }^{11,82,84,85}$.

Surface states are also generally polar and the polarity in the wall strongly interacts with surface polarity near the intersection between the domain wall and the surface. Furthermore, energy minimisation requires the strain compatibility between surface strains and the strains due to the domain walls. The initial conjecture that this would lead to funnel-like profiles was shown to be wrong and two characteristic excitations were found ${ }^{86}$. These are long-wavelength relaxations in the surface layer and a 'pinching' movement of the domain wall, i.e., domain walls slightly narrower near the surface. LEEM is particularly sensitive to such surface effects ${ }^{87,88}$ and enables characterisation of polarisation profiles at the intersection of domain walls with the surface $^{33,83}$. The LEEM contrasts on the surface arise from polarisation charges, resulting from the polarisation inside domain walls, which deviate the incoming low energy electrons ${ }^{33,83}$. Injection of higher energy electrons permits screening these charges and removing the contrast, which can be recovered by moderate annealing at $330^{\circ} \mathrm{C}$ (FIG. 3f).

Despite the recent progress in the characterization of polar domain walls in non-polar materials, the experimental demonstration of the bi-stable polarity of domain walls and its switching has not yet been realised. It remains the most challenging task in the field of polar domain walls in both ferroelectric and non-polar materials, and a crucial step needed towards their potential use as functional elements. Another critical aspect will be a detailed understanding of the control of the walls' position and motions by means of electric or elastic stress fields. The effect of electric fields on ferroelastic wall motions ${ }^{89,90}$ and more generally the control of domain wall dynamics (BOX 2) will be the key.

\section{Conductive domain walls in ferroelectrics}

Enhanced electrical conductivity in ferroelectric domain walls has been reported in several materials, but we are just beginning to understand the underlying nanoscale physics. To reflect the state of the art, we discuss here key observations material by material, highlighting the specificities for each case. The established fundamental mechanisms that lead to (or co-determine) domain wall conduction in all these materials are summarized in BOX 3.

\section{Bismuth ferrite}

Bismuth ferrite $\left(\mathrm{BiFeO}_{3}\right)$ holds a special place in the history of research on functionally active domain walls: wall conductivity, and even superconductivity, had been reported in previous studies ${ }^{91-93}$ on copper chlorine boracite $(\mathrm{Cu}-\mathrm{Cl}-\mathrm{B})$ and non-stoichiometric tungsten trioxide $\left(\mathrm{WO}_{3-\mathrm{x}}\right)$, but the first spatially resolved measurements explicitly demonstrating locally enhanced conduction, using conductive atomic force microscopy 
(c-AFM), were collected from $109^{\circ}$ and $180^{\circ}$ domain walls in the rhombohedral phase of $\mathrm{BiFeO}_{3}$ (FIG. 5a) ${ }^{94}$. A couple of years later, observations of conduction in $71^{\circ}$ walls followed in different sets of thin film samples ${ }^{95-97}$.

Early density functional theory (DFT) based calculations ${ }^{98}$ suggested that the relative changes in conduction between domain walls and bulk, and among the different domain wall types, were associated with a fundamental alteration in the local electronic band structure: models showed that conduction bands were depressed in energy at the walls, while there were only very minor shifts in valence band edges (FIG. 4b). This notion was supported by experiment: scanning tunnelling spectroscopy measurements ${ }^{97}$ were used to directly probe the density of states as a function of location (traversing both $109^{\circ}$ and $71^{\circ}$ walls). The errors in band edge energy determination were significant, but a noticeable dip in the energy of the conduction band edge at the $109^{\circ}$ domain wall was seen, while meaningful signs of valence band edge changes were not. These reports pointed to observations of conductivity being the result of local changes in band structure (and reduced band-gaps) at the domain walls. However, it has been contested as to whether these local changes are significant enough to cause the increase in conductivity observed ${ }^{99}$.

Attempts to elucidate fundamental band structure alterations through direct currentvoltage measurements have often been deeply frustrated by the two-probe geometry inherent to the c-AFM technique: conduction has consistently been found to be dominated by the barrier resistance between the tip and the material, with both Schottky and Fowler-Nordheim behaviour being claimed ${ }^{95,96,100}$. It has been suspected, particularly in work by Farokhipoor and Noheda ${ }^{95,96}$, that barrier height reductions occur because of enhanced oxygen defect concentrations at the domain walls ${ }^{101}$ (FIG. 4a); Maksymovych et al. ${ }^{102}$ acknowledged that such vacancies should certainly be present, but consider that the multistate resistance levels induced under applied fields, in their work, had response times deemed too rapid to be caused by oxygen vacancy migration. Instead, they proposed that microscopically reversible distortion of the polarisation structure at the domain wall, induced by the voltage applied to the cAFM tip during the measurement, might have been responsible for changing the conduction behaviour. Polarisation discontinuities and associated strong local electric fields have also been seen to play a role (FIG. 4c) and complex polarisation patterns have been observed using high-resolution STEM ${ }^{103}$. Furthermore, c-AFM measurements on written domain structures, designed to generate abutting polarisation patterns, have shown that "head-to-head" walls (FIG. 4c) conduct while "tail-to-tail" walls (FIG. 4c) do not ${ }^{104}$. In addition, it has been recently argued that strain-compatible domain walls in $\mathrm{BiFeO}_{3}$ localize free excess electrons and create intrinsic polaronic ingap states ${ }^{105}$. This mechanism might dominate the thermally activated domain-wall conduction, e.g., in thin film materials with a high density of domains walls.

An overriding observation is that $\mathrm{BiFeO}_{3}$ research has always been plagued by uncontrolled and often uncharacterised point defects: the presence of the transition metal $(\mathrm{Fe})$ and the associated degree of freedom in valence state readily facilitate nonstoichiometry. Rojac et al. ${ }^{106}$ used STEM techniques to reveal significant concentrations of $\mathrm{Fe}^{4+}$ ions at $\mathrm{BiFeO}_{3}$ domain walls, resulting from the accumulation of bismuth vacancies. Mixed $\mathrm{Fe}^{3+}-\mathrm{Fe}^{4+}$ valence states offer possibilities for conduction through electron hopping, suspected as key in conductivity measurements performed by Lee et $a l .{ }^{107}$. Perhaps the most compelling evidence for the dominant role of defect accumulation in generating enhanced conductivity at domain walls in $\mathrm{BiFeO}_{3}$ can be 
found in the work of Stolichnov et al. ${ }^{108}$ : they found that, when conducting domain walls were moved using applied electric fields, enhanced conduction persisted where the domain walls had been, prior to field-induced movement, indicating that the sheet conduction and the wall could be decoupled.

Actually, the enhanced electrical conduction in $\mathrm{BiFeO}_{3}$ domain walls is likely the result of all the mechanisms discussed in FIG. 4, making it both a complex and versatile system. Going beyond just enhanced conductivity, magnetotransport ${ }^{107,109-111}$ was studied at domain walls in $\mathrm{BiFeO}_{3}$, expanding possible domain wall applications into the realm of spintronics.

\section{Hexagonal manganites}

A breakthrough concerning the disentanglement of conduction contributions was the discovery of anisotropic transport properties at ferroelectric domain walls in hexagonal (h-) manganites $\left[R \mathrm{MnO}_{3}(R=\mathrm{Sc}, \mathrm{Y}, \mathrm{In}, \mathrm{Dy} \text { to } \mathrm{Lu})\right]^{112-114}$. Hexagonal manganites exhibit uniaxial (improper) ferroelectricity and naturally develop all fundamental types of $180^{\circ}$ domain walls, that is, neutral side-by-side, negatively charged tail-to-tail and positively charged head-to-head walls (FIG. 5c). The walls are stabilized by topologically protected six-fold anchor-points ${ }^{112,115}$, which prevents the material from forming, e.g., simple stripe-like domains with neutral walls. In 2012, Meier et al. performed local transport measurements and showed that the conductivity at the charged domain walls originates from bound charges and the related electrostatic potential, locally modifying the electronic band structure (intrinsic) ${ }^{113,116,117}$, analogous to the scenario depicted in FIG. 4c. It is a typical example where the wall conductivity depends on the crystallographic orientation of the domain wall normal.

At tail-to-tail domain walls, the mobile majority carriers (holes) feel the pronounced electrostatic fields from the negative bound charges, leading to hole accumulation and, hence, enhanced hopping conductance (p-type). Turner et al. revealed that the associated carrier mobilities are surprisingly high, in the order of $100 \mathrm{~cm}^{2} \mathrm{~V}^{-1} \mathrm{~s}^{-1}$ at room temperature, and amongst the highest reported in oxide systems ${ }^{118}$. Accordingly, the positive bound charges at head-to-head domain walls are screened by hole depletion, leading to reduced conductance. Recently, it was observed that head-to-head domain walls can also deliver enhanced conduction over bulk, but only under higher electric fields ${ }^{31}$. It is clear that, in this case, the active carriers are n-type ${ }^{31,118}$. Such carriers arise from a significant band bending with the conduction-band minimum dipping below the Fermi energy, which leads to the formation of an electronic inversion layer $^{31}$ (FIG. 4c). Thus, the origin of conduction at charged domain walls in hexagonal manganites is well-understood. At the quantitative level, however, there are still some discrepancies between the calculated and measured carrier densities and their participation in transport ${ }^{31,118}$. Such discrepancies likely arise from variations in the domain-wall charge state "hidden" within the bulk ${ }^{119}$ and secondary contributions due to interactions between the domain walls and point defects.

In contrast to the charged domain walls, neutral side-by-side domain walls in $\mathrm{h}-\mathrm{RMnO}$ (FIG. 5c) show a similar electronic structure as the bulk ${ }^{120}$. Their transport properties are extrinsic and arise from the complex defect chemistry at domain walls, ranging from insulating $^{112}$ to conducting ${ }^{121}$ (FIG. 4a). For example, it has been demonstrated that oxygen interstitials have a propensity to form at neutral domain walls ${ }^{32}$, giving rise to enhanced DC conduction (p-type) and increased AC conductivity at frequencies in the 
kilo- to megahertz range ${ }^{32}$. Higher drive frequencies in the terahertz regime lead to dynamical domain-wall displacements, resulting in anomalous microwave AC conductivity at neutral domain walls ${ }^{122}$.

\section{Barium titanate}

Another ferroelectric where the high conductivity is explained within the scenario of FIG. 4c is $\mathrm{BaTiO}_{3}$. In this system, Sluka et al. have used macroscopic current measurements to show that, at head-to-head domain walls, the static conductivity can increase by 9 orders of magnitude ${ }^{123}$. These strongly charged domain walls are more difficult to obtain than in improper ferroelectrics, like hexagonal manganites, because of their high energy $\operatorname{cost}^{124}$, but they are stabilized by the strain-compatibility of the adjacent ferroelastic domains and an almost perfect compensation of the bound polarisation charges by free carriers, which are at the origin of the high conductivity. In $\mathrm{BaTiO}_{3}$, and ferroelectrics in general, such compensation cannot be provided by the free carriers readily available in the material but originates from electron transfer across the bandgap or external charge injection. At low voltages $(<8 \mathrm{~V})$, the free carriers in the wall are separated from the electrode by an insulating gap due to the flattening of the electric potential profile by the equipotential electrodes and large voltages are required to observe the electron transfer and an increase in conductivity ${ }^{123}$. At tail-totail walls, experiments show that the conductivity is like the bulk values, which might arise from electron depletion ${ }^{124,125}$. Also, oxygen vacancies that are screening tail-totail walls, in particular in samples with high oxygen deficiency ${ }^{126}$, exhibit a low mobility compared to electrons and hence lead to a low conductivity.

\section{Lithium niobate}

Thus far, the higher static conductivity of domain walls in the uniaxial ferroelectric lithium niobate $\left(\mathrm{LiNbO}_{3}\right)$, observed in an early study of Aristov et al. ${ }^{127}$, has been attributed to partially charged domain walls, analogous to the scenario depicted in FIG. 4c, but where the domain walls are inclined by a few degrees instead of being vertical. On a macroscopic scale, Schröder et al. have shown using c-AFM on both faces of single crystals that domain walls in $\mathrm{LiNbO}_{3}$ are inclined with respect to the ferroelectric polarisation direction and that this inclination can be controlled by a change in dopant concentrations, e.g., magnesium (inclination up to $\left.0.3^{\circ}\right)^{128}$. The inclination, confirmed by Čherenkov second-harmonic generation measurements (FIG. 5d $)^{129,130}$, results from an incomplete poling of the material and can be much larger in sub-micrometre thick samples (inclination up to $\left.20^{\circ}\right)^{27,131}$. Similarly, inclined domain walls have also been observed in $\mathrm{LiTaO}_{3}$ by introducing a composition gradient along the polarisation axis, which changes the temperature of the transition to the paraelectric phase and the coercive field ${ }^{132,133}$. Such a strategy should be successful in $\mathrm{LiNbO}_{3}$ as well but has not been demonstrated yet.

Inclined domain walls lead to partial head-to-head configurations with an accumulation of free electronic carriers, leading to an intrinsic rise in conductivity ${ }^{134}$. The LandauGinzburg-Devonshire theory predicts an increase of conductivity by one order of magnitude at small angles $\left(0.07^{\circ}\right)^{134}$, which could be obtained in the first experiments only with additional carriers provided by illumination at an energy larger than the bandgap $^{128}$. The predicted conductivity across tail-to-tail walls, which should be at least an order of magnitude smaller than the one for head-to-head walls due to the low mobility of holes compared to electrons ${ }^{134}$, has not been observed experimentally yet. It was 
realised later by Eng and Gruverman that, close to the surface, there is an additional bending of the upper segment of the wall, away or toward the polarisation direction, which can be controlled by the application of sub-coercive voltage (FIG. 5d - right inset $)^{131,135}$. This surface effect leads to strong variations in carrier density in the first $100 \mathrm{~nm}$ and dominates the overall conductivity measured by c-AFM, which can then be tuned by 2 orders of magnitude ${ }^{131,135}$. Similar domain wall bending is expected in most ferroelectrics but has not been investigated yet. Additional free carriers that are excited while probing the walls with c-AFM can further give rise to large AC conductivity ${ }^{136}$.

In fact, the conductivity at domain walls is likely to be even higher than the value obtained through these c-AFM measurements (conductance $\sim 10 \mathrm{pS}$ ) where substantial contact barriers at the tip lead to non-ohmic behaviour ${ }^{128,137}$. As such, the work of Werner et al. ${ }^{137}$ is very instructive. It shows a 4 orders of magnitude difference between the current mapped with c-AFM $(\sim 1 \mu \mathrm{A})$ and the current recorded through macroscopic electrodes contacting several domain walls $(\sim 10 \mathrm{~mA})$, leading to a real enhancement of the conductivity at domain walls by 13 orders of magnitude compared to that of the bulk.

On the nanoscale, Gonnissen et al. have shown with STEM that domain walls in $\mathrm{LiNbO}_{3}$ are not straight but exhibit meanders and kinks, which result in local head-tohead or tail-to-tail sections where bound charges accumulate (FIG. 5d - left inset). Compared with $\mathrm{PZT}^{7}$ (FIG. 5b), these sections are spatially less extended $(<10 \mathrm{~nm})$, but it has been argued that the charges could be screened by stabilized defects ${ }^{138,139}$, which could play an extrinsic role in the enhanced conductivity of $\mathrm{LiNbO}_{3}$. Further work is highly desirable to investigate this effect.

\section{Lead zirconate titanate}

Another system that underlines the complexity of domain-wall related transport phenomena in ferroelectrics is PZT. In 2008, Jia et al. showed that, at the atomic scale, both neutral side-by-side and charged head-to-head $180^{\circ}$ domain walls arise in $\mathrm{SrTiO}_{3} / \mathrm{Pb}\left(\mathrm{Zr}_{0.2} \mathrm{Ti}_{0.8}\right) \mathrm{O}_{3} / \mathrm{SrTiO}_{3}$ trilayers ${ }^{7}$. In particular, they observed that domain walls exhibit local head-to-head sections, where the wall tilts away from the ideal neutral structure (FIG. 5b). As discussed in the previous sections on, e.g., hexagonal manganites and $\mathrm{LiNbO}_{3}$, this tilting results in domain wall bound charges that require screening (FIG. 4c), for example, by oxygen vacancies (extrinsic) or electronic carriers (intrinsic), possibly stabilized by a local change in the $\mathrm{Ti}$ valence state from $\mathrm{Ti}^{4+}$ by $\mathrm{Ti}^{3+}$ $\left(\mathrm{REF}^{7,140}\right)$. These excess electrons on $\mathrm{Ti}$ sites, which experience a strong on-site Coulombic repulsion, could take the form of localized small polarons ${ }^{141}$.

The formation of domain walls with neutral and head-to-head sections later became the key to understand the thermally activated transport at $180^{\circ}$ walls in simple $\mathrm{Pb}\left(\mathrm{Zr}_{0.2} \mathrm{Ti}_{0.8}\right) \mathrm{O}_{3}$ thin films ${ }^{142-144}$. Guyonnet et al. proposed that the charged sections exhibit an increased defect density (oxygen vacancies), providing trap states for hopping mediated transport along walls (FIG. 4a) ${ }^{142}$. To enable extrinsic defectmediated conduction, a certain threshold density of oxygen vacancies is to be reached, as the same team demonstrated by controlling growth conditions and atmosphere ${ }^{145}$. Here, the highly localized electric fields, which are used to create the domain walls with c-AFM, come into play. Such fields may drive the reorganization/injection of oxygen vacancies, which then preferentially segregate to newly formed walls. Additional free 
carriers that are excited while writing the walls with c-AFM can further enhance the DC conductance or, at high frequencies (gigahertz), oscillate between barriers, giving rise to large $\mathrm{AC}$ conductance $(\sim 100 \mathrm{nS})^{146}$.

Even higher, metallic conductance, was achieved by writing nanodomains with strongly curved walls and, hence, a large fraction of charged segments ${ }^{147}$. The work on strongly curved walls showed that both electron tunnelling and metallic conductivity play a role in PZT and confirmed the strong correlation between domain wall conduction and curvature - a concept that was then expanded to nominally neutral $90^{\circ}$ domain walls ${ }^{7}$ : by strain engineering, $90^{\circ}$ domain walls in PZT were forced to bend away from their mechanically compatible position, inducing partially charged walls with metallic-like conductivity ${ }^{148,149}$. Importantly, the $90^{\circ}$ domain walls were generated at $\mathrm{T}=4 \mathrm{~K}$, that is, a temperature at which defects cannot migrate, which led to the conclusion that the conduction arises from intrinsic band bending effects (FIG. 4c). More recently, stateof-the-art microscopy studies provided additional insight, revealing the emergence of complex Néel-like polarisation rotations (FIG. 1f) across domain walls in PZT, which can locally violate charge neutrality and promote domain wall conductance ${ }^{55-57}$.

The research on PZT highlights the importance of domain wall bound charges for conductivity to arise (see FIG. 4c). However, the charged sections are usually "hidden" within the bulk so that it is challenging to establish a quantitative relation between their density and the domain wall conduction. In addition, beyond walls with charged sections, more complex polarisation rotations emerge, and both intrinsic and extrinsic contributions appear to play role for the domain wall conduction, critically depending on small variations in the domain wall orientation and growth conditions.

In summary, the electronic transport at domain walls is studied intensively. So far, however, most application oriented studies focus on controlling the domain wall behaviour via electric fields and defect densities; other intriguing phenomena like the interaction of light with domain walls is still a rather overlooked topic that has only been studied in $\mathrm{LiNbO}_{3}{ }^{128}$ and $\mathrm{BiFeO}_{3}{ }^{17,111,150-153}$. Further studies, taking advantage of near-field techniques, e.g., tip-enhanced Raman spectroscopy, which overcome the diffraction limit and give the possibility to probe domain walls with a reduced bulk contribution, are desirable ${ }^{154}$. Another promising field that deserves further attention concerns the local interaction of electric and magnetic degrees of freedom, leading to unusual correlation phenomena ${ }^{155}$ and domain wall magnetotransport ${ }^{107,109-111}$.

\section{Combining domain walls and transition regions for emerging properties}

\section{Beyond conductivity and polarity at domain walls}

From a more conceptual point of view, domain walls represent transition regions of the order parameter. As such, they can exhibit a wide range of unusual properties, going way beyond the changes in conductivity and polarity discussed in the previous sections. For example, at simple Ising-type walls (FIG. 1d), the polarisation is suppressed and the free energy exhibits a local maximum with a negative curvature ${ }^{156}$. This specific profile of the free energy across a domain wall, predicted by Landau theory, has been observed experimentally through a precise mapping of polarisation and local electric fields in a $\mathrm{SrTiO}_{3} / \mathrm{PbTiO}_{3}$ superlattice ${ }^{156}$. As a direct consequence of the unusual curvature of the energy landscape, the walls exhibit negative permittivity. The effect 
can be boosted by engineering dielectric/multidomain ferroelectric superlattice, leading to a situation where the negative domain wall permittivity dominates the macroscopic response of the system ${ }^{157}$. The approach allows for designing superlattices with improved permittivity, which is higher than for the individual constituent dielectric $\left(\mathrm{SrTiO}_{3}\right)^{156,158}$, enabling a new generation of low-energy field-effect transistors ${ }^{159}$.

Furthermore, at the atomic scale, transition regions of the order parameter correlate with structural changes, altering interatomic distances and, hence, the electronic bonds and the local strain state ${ }^{160}$. Such structural inhomogeneities associated with domain walls have a direct impact on the propagation of phonons. Royo et al. ${ }^{161}$ have demonstrated that domain walls can act as phonon polarizers and filter phonons depending on their polarisation ${ }^{161}$. In the model case of tetragonal $\mathrm{PbTiO}_{3}$, they demonstrate that $180^{\circ}$ domain walls scatter phonons associated with atomic displacements along the direction transverse to a heat flux, while phonons associated with atomic displacements along the longitudinal direction are transmitted ${ }^{161}$. Thus, a single domain wall can lead to a reduction of the thermal conductance by $\sim 43 \%$, essentially through the scattering of low-frequency phonons. Beyond changes in thermal conductance, such a domain-wallbased phonon polarizer could find applications in devices relying on nonradiative energy relaxation and inter-band recombination processes, which are governed by polarisation-dependent selection rules.

Strain gradients at domain walls can also lead to the emergence of chemically novel 2D-phases that have not yet been synthesised by standard chemical routes and remain a specificity of domain walls ${ }^{162}$. Such unusual chemical phase has been reported at the ferroelastic domain walls of multiferroic orthorhombic $\mathrm{TbMnO}_{3}$ thin films ${ }^{162}$. It arises from the local substitution of $\mathrm{Tb}$ atoms by $\mathrm{Mn}$ atoms on almost all atomic sites. Remarkably for applications in spintronics ${ }^{163}$ and magnonics ${ }^{164}$, the corresponding local increase in Mn content changes the local magnetic properties and leads to a positive in-plane magnetic moment per atomic plane parallel to the domain wall, while the domains are antiferromagnetic ${ }^{162}$. Another example taking advantage of a different chemistry at domain walls is the 'nano-capacitors' engineered by defect-chemistry in strained $\mathrm{SrMnO}_{3}$ thin films ${ }^{165}$. A comparison of c-AFM conductance maps with SHG imaging measurements reveals electrically decoupled conducting nanoarrays of polar domains delimited by insulating walls, which can be individually charged. Complementary density functional theory calculations indicate that the walls are insulating and act as a barrier to conduction because of the accumulation of oxygen vacancies driven by local strain ${ }^{165}$.

Thus, the transition region of order parameters associated with domain walls is highly relevant both from a fundamental as well as a technological point of view. This has also been realised by other communities, which is currently leading to an expansion into adjacent research fields with intriguing synergetic effects. As such, there has been a strong interest in properties of phase boundaries, homo- and heterointerfaces, and other quasi-2D objects, all described here-after as transition regions.

\section{Transition regions}

One example of a conceptually new type of transition region that falls into this category is the epitaxial trilayer heterostructure $\mathrm{PZT} / \mathrm{La}_{0.7} \mathrm{Sr}_{0.3} \mathrm{MnO}_{3} / \mathrm{PZT}$, which can be described as an homogeneous artificial material with inversion-symmetry properties ${ }^{166}$. Indeed, the interlayer ( $\mathrm{La}_{0.7} \mathrm{Sr}_{0.3} \mathrm{MnO}_{3}$ ) offers the possibility to switch the polarisation 
direction of the top PZT layer without changing the polarisation direction of the bottom PZT layer. When the polarisations of the two PZT layers are antiparallel, the material exhibits an inversion symmetry while when they are parallel, the inversion symmetry is broken. Such trilayer systems can thus act as nano-sized light emitters, where a SHG output signal - correlated with the loss of inversion symmetry - can be controlled by applying a voltage ${ }^{166}$. Subsequent experiments have demonstrated the generality of this approach, which is robust against an exchange of the control ferroelectric layers as well as the oxide interlayer. The strategy opens the possibility to alter other properties of the interlayer or its interfaces and transfer what has been learnt from charged head-to-head and tail-to-tail walls to thin film heterostructures and multilayers. To optimize such structures, the control of interfaces will be critical and could rely on the accumulated knowledge acquired through the study of multiferroic heterostructures. Here, ionic, electronic, strain and exchange couplings at interfaces result in transition regions, often down to a couple of atomic layers only, both in the ferroelectric and ferromagnetic materials $^{167-174}$.

Symmetry breakings have also been observed in homogeneous thick films ${ }^{175,176}$. In this regard, the recent discussion in a work of Noheda on so-called "transitional ferroelectrics" is appealing ${ }^{176}$. The denomination comes from the observation of a gradual change of structure from a tetragonal symmetry at the top of a thick film of $\mathrm{BaTiO}_{3}$ to an orthorhombic symmetry at the bottom ${ }^{176}$. The transition region between both phases is characterized by a reduced symmetry where the polarisation rotation is coupled to the strain gradient (FIG. 6a). The ease with which the polarisation rotates in this transition region under small electric fields leads to huge and temperatureindependent dielectric responses (> 4000) and large piezoelectric responses ${ }^{176}$. These exceptional properties arise from the unusual characteristics of the transition region, which is not a polycrystal but also not a single crystal, not a relaxor but also not a homogeneous ferroelectric.

A remarkably similar observation has been reported in free standing single-crystal thin membranes of $\mathrm{BaTiO}_{3}$. In these systems, under increasing stress due to the mechanical bending of the membrane, the tetragonal domains evolve from $c$-domains (out-of-plane) to $a$-domains (in-plane), with a continuous rotation of the dipoles between both domains (FIG. 6b) ${ }^{177}$. This transition region where the dipoles rotate is thought to be at the origin of the exceptionally large flexibility and super-elasticity (recoverable strain $\sim 10 \%$ ) of these membranes since it could eliminate most of the mismatch stress between domains ${ }^{177}$. Also, it resembles the transition region discussed in "transitional ferroelectrics" but with tetragonal nanodomains as building blocks, instead of a phase with reduced symmetry.

It is highly likely that these transition regions will additionally exhibit unusual evolutions with temperature. In PZT, ab-initio calculations have been used by Nahas et al. to characterize the evolution of the ferroelectric domain structure with temperature $^{178}$. On heating, an initial labyrinthine domain pattern, which consists of convoluted stripes and meandering domains, evolves into a less-symmetric parallelstripe state before to undergo the transition to the more-symmetric paraelectric phase (FIG. 6c). The demonstration of such an inverse transition ${ }^{179}$, i.e., a transition to a lesssymmetric domain state on heating, is a first in ferroelectric oxides and is in agreement with the experimental evolution of domains in $\mathrm{BiFeO}_{3}$ upon cooling from different high temperatures ${ }^{178}$. 
The remarkable communality between the different transition regions described above and their evolution with driving fields (temperature, stress) is that they lead to exceptional properties and coupling phenomena. Also, it is highly likely that they can be triggered by light and will react to mechanical strain, and thus provide interesting piezo-photovoltaic properties ${ }^{180}$. The existence of such regions relates to different origins or driving forces, ranging from chemistry, phase coexistence, frustrations, fluctuations, and chiral textures, all of which can be in principle encountered in or around domain walls. Therefore, most of these transition regions probably exist in the vicinity of domain walls, at a smaller length scale, and shall receive more attention to foster mutually beneficial research. The fact that the inverse transition described previously finds its origin in the diffusion and relaxation of topological defects localized at the junction of different domains ${ }^{178}$ is a striking example of the possibilities offered by such cross-fertilisation. One could also ask if the recent superconductivity observed in an "infinite-layer" nickelate of specific chemistry ${ }^{181}$ could be observed as well in the domain walls of rare-earth nickelates, which constitute 2D objects potentially stabilizing defects, and have been less studied than in other oxides ${ }^{182}$. No doubts that superconductivity, super-elasticity, giant dielectric properties at domain walls are in reach.

\section{Outlook}

For future investigations, we believe that insights into the full 3D structure of the domain walls, down to the nanoscale, are highly desirable in order to answer open questions about the microscopic origin of the intriguing functionalities. Experimental techniques, such as dark-field X-ray microscopy, which can map lattice distortions around domain walls in bulk samples ${ }^{183}$, or 3D atom-probe tomography, which could provide access to the chemical identity of individual atoms at domain walls ${ }^{184}$, would be relevant. In particular, they might help to understand the role of point defects interacting with domain walls and disentangle extrinsic and intrinsic effects to gain reliable quantitative information. The latter is important when moving towards domainwall based devices, where well-defined and deterministic behavior is critical.

Regarding applications, the standard idea is to create, move and eliminate domain walls with an external field to control the functionality. This approach, however, relies on classical device geometries, e.g., where conductive domain walls are used as interconnects between electrodes. A conceptually new approach is to keep the walls static and use their versatile properties to control electronic signals. Going beyond just conductance, by driving, for example, metal-insulator transitions or magneto-resistive changes within domain walls represents a promising pathway. Different domain walls could emulate the behavior of different electronic components ${ }^{31,32}$, representing the building blocks of domain-wall based networks and circuitry, where the atomic-scale dimension associated with the domain walls is fully taken advantaged of instead of using them as active components in much larger devices. With this, we believe that domain walls could play a key role in the transition from nano- to atomic scale electronics.

\section{Acknowledgements}

GFN thanks the Royal Commission for the Exhibition of 1851 for the award of a 
Research Fellowship. JK and MG acknowledge financial support from the Fond National de Recherche Luxembourg through a PEARL Grant (No. FNR/P12/4853155/Kreisel). JH acknowledges financial support from the Czech Science Foundation (project no. 19-28594X). DM was supported by the Research Council of Norway through its Centres of Excellence funding scheme, project number 262633, "QuSpin" and by NTNU via the Onsager Fellowship Program and the Outstanding Academic Fellows Program.

\section{Author contributions}

All authors have read, discussed and contributed to the writing of the manuscript.

\section{Competing interests}

The authors declare no competing interests. 


\begin{tabular}{l}
\hline Box 1 Topological defects in ferroelectrics \\
\hline A topological defect is a localized discontinuity of the order parameter that cannot \\
be removed by a local continuous change in the order parameter configuration ${ }^{11,185}$. \\
When localized in one direction but extended in the two other directions, e.g., as a \\
domain wall, it is a planar defect. The line separating regions of opposite polarities \\
within a domain wall, e.g., in a Bloch wall, is a line defect (FIG. 2a) ${ }^{9,10}$. Domain \\
walls, as well as line defects within domain walls, are not the only topological defects \\
that attract the attention of the ferroelectrics' community. Inspired by magnetic \\
textures, researchers explore now properties of other non-trivial ferroelectric \\
structures - often described as topological defects - consisting of arrays of individual \\
polarisation flux closure vortices (FIG. 2 b) $)^{186}$, polar-skyrmion bubble domains \\
(FIG. 2 c) ${ }^{187}$ and perhaps also ferroelectric Bloch skyrmions ${ }^{188}$, that could be the most \\
direct counterparts of the magnetic Bloch skyrmions known from thin films of chiral \\
magnets ${ }^{189}$. \\
In thermally activated systems, simulations reveal highly mobile polar vortices ${ }^{190}$. \\
Their lifetime is short, and these vortices are prone to be destroyed by applied electric \\
fields ${ }^{190}$, except when they are attached to domain walls, surfaces or defects ${ }^{191,192}$. \\
Most intriguingly, junctions between domain walls and kinks in domain walls also \\
generate polarisation vortices ${ }^{76,193,194}$. Under motion of the wall, such vortices are \\
expected to generate a magnetic field in nonmagnetic materials by rotating polar \\
vectors inside domain walls ${ }^{195}$. Experimentally, only very weak magnetic signals \\
have been observed ${ }^{196}$ and more research is urgently needed.
\end{tabular}




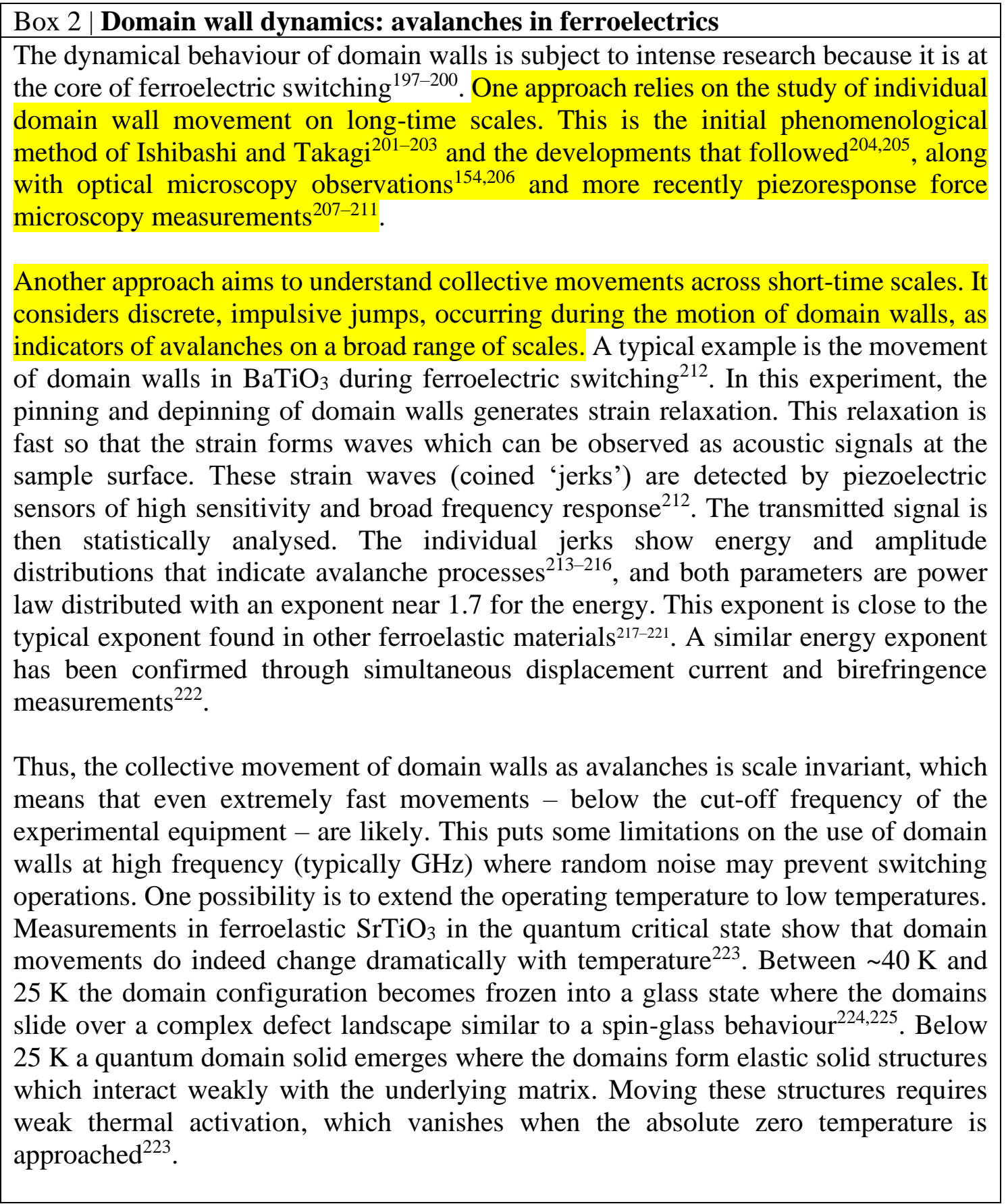




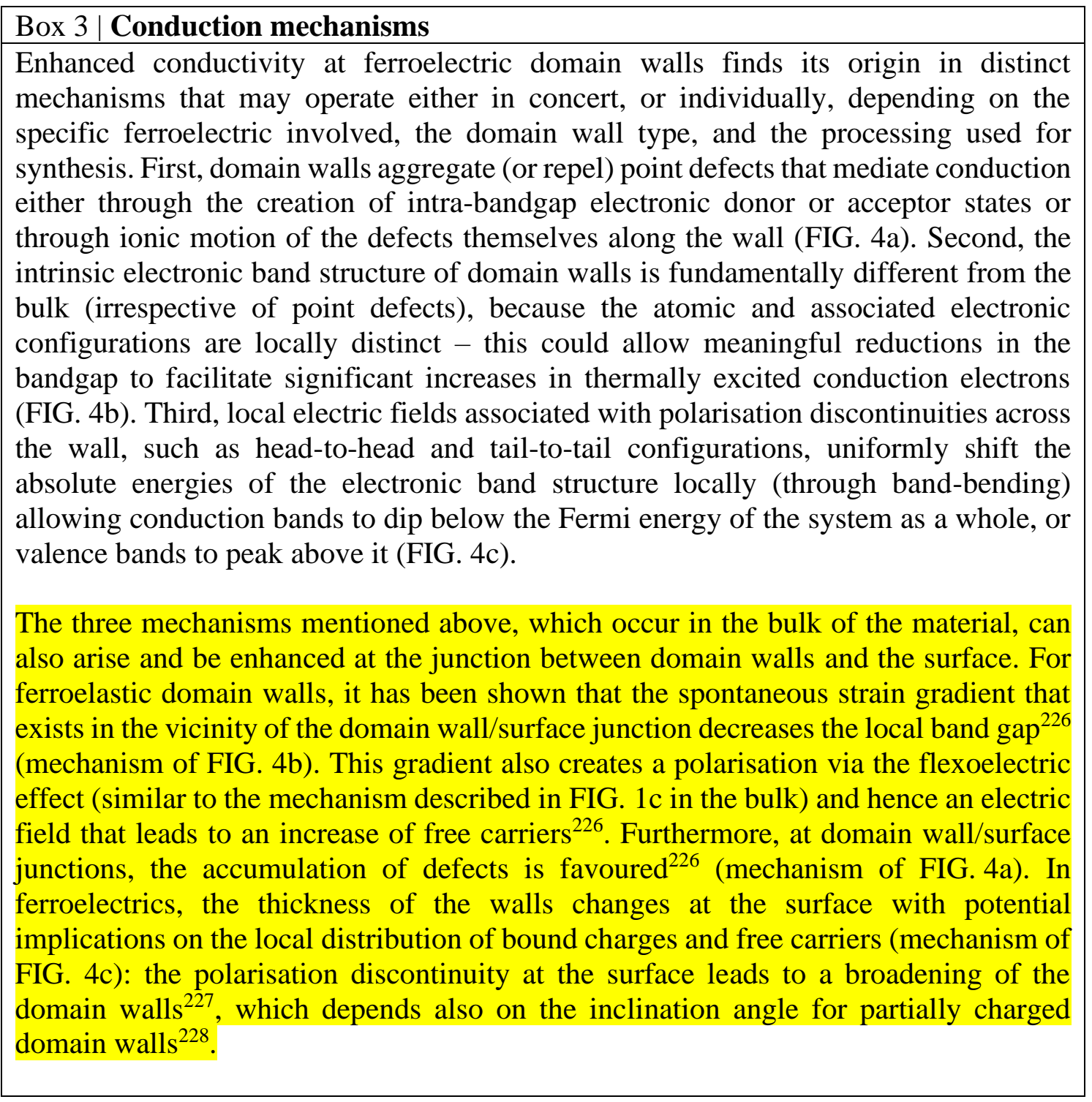


a One order parameter

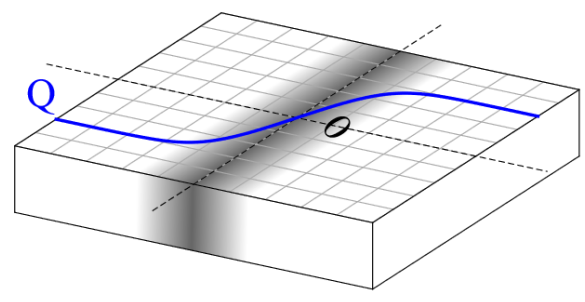

b Emerging order parameter

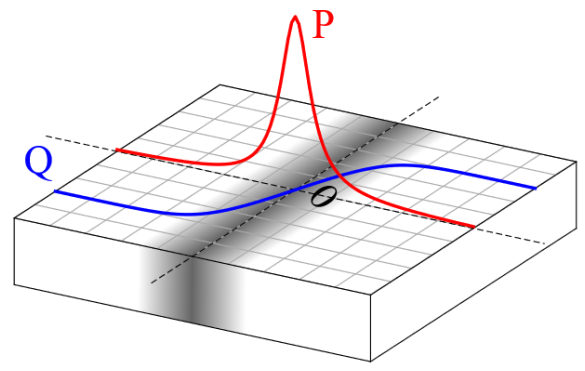

c

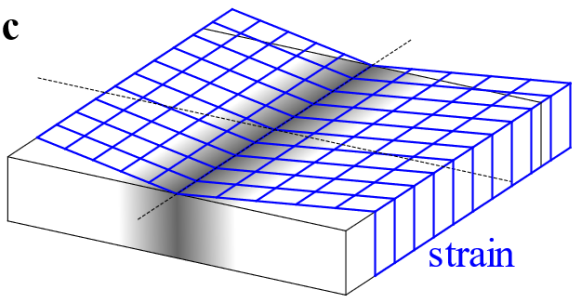

Ferroelectrics

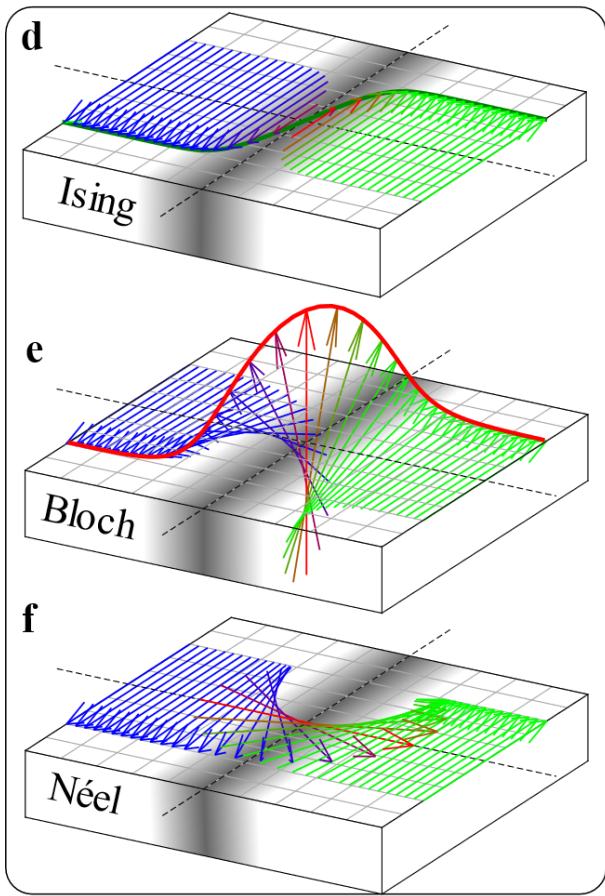

Fig. 1. Mechanisms leading to polar domain walls. a | Schematic of an elementary domain wall with a single order parameter and tanh profile. $\mathbf{b}$ | Phase transition in a domain wall, with the emergence of an additional instability, which may be a polarisation component $P$. c | Sketch of a ferroelastic wall, with a discontinuity (or gradient) in spontaneous strain. d | Ferroelectric elementary Ising wall. e | Ferroelectric Bloch wall, where the polarisation rotates in the plane of the wall. $\mathbf{f} \mid$ Ferroelectric Néel wall. 
a Ising line defect

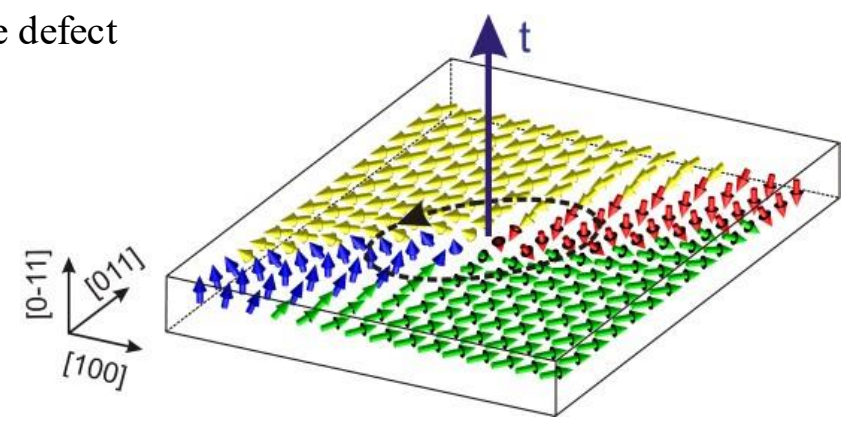

b Polarisation flux closure vortex

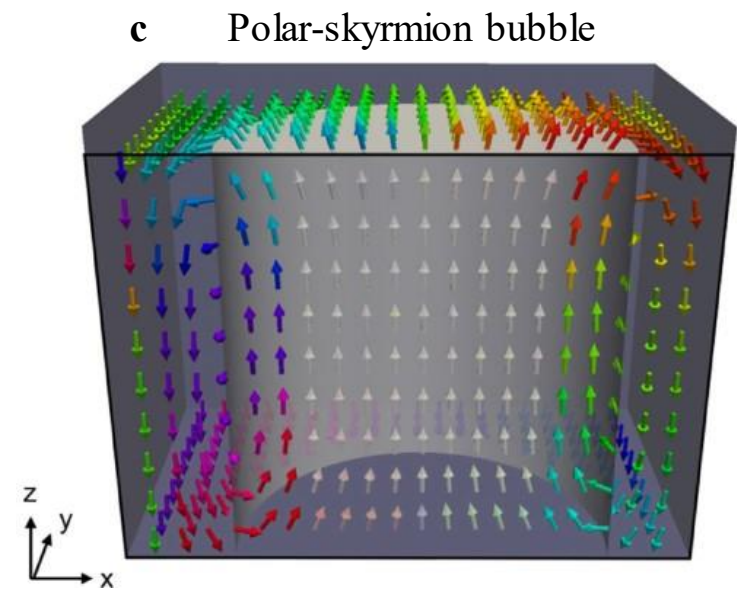

Fig. 2. Examples of topological defects in ferroelectrics beyond domain walls. a Ising line defect in rhombohedral $\mathrm{BaTiO}_{3}$ as obtained from the model described in REF. ${ }^{10}$. When circumventing the core of this defect following a closed path (dashed line) around its axis $t$, the polarisation on this path rotates around the [-211] axis, which is perpendicular to $t$. Reproduced with permission from REF. ${ }^{10}$. b | STEM polar displacement vector map of a single vortex in a $\left(\mathrm{SrTiO}_{3}\right)_{10} /\left(\mathrm{PbTiO}_{3}\right)_{10}$ superlattice. Reproduced with permission from REF. ${ }^{186}$. $\mathbf{c}$ | Three-dimensional cross-sectional view of a polar-skyrmion bubble. Reproduced with permission from REF. ${ }^{187}$. 

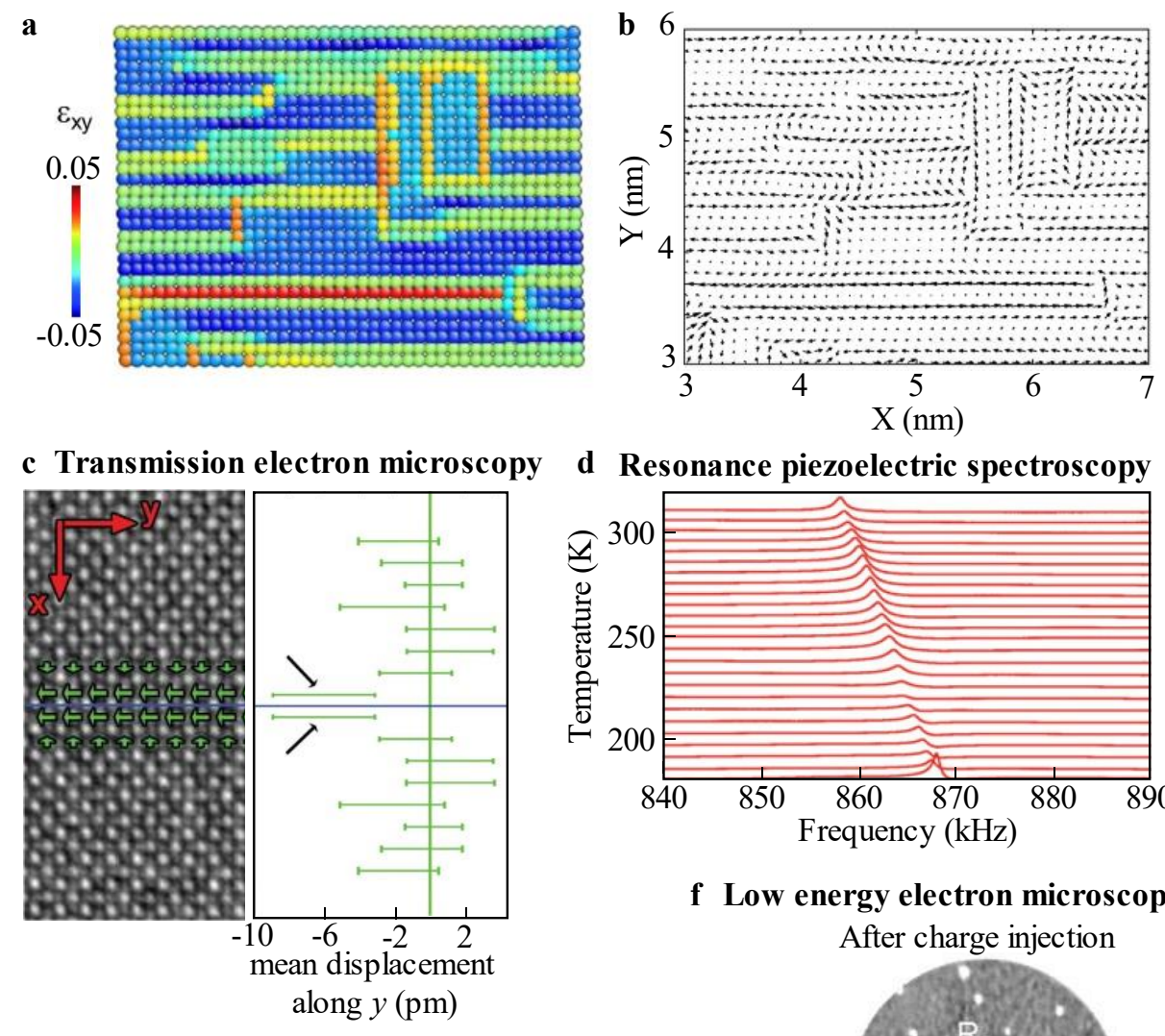

d Resonance piezoelectric spectroscopy

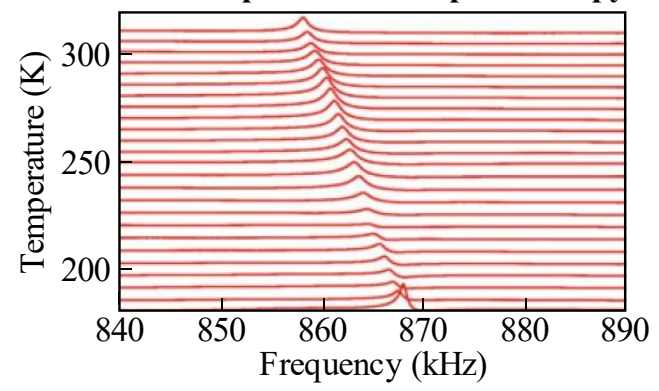

e Second harmonic generation

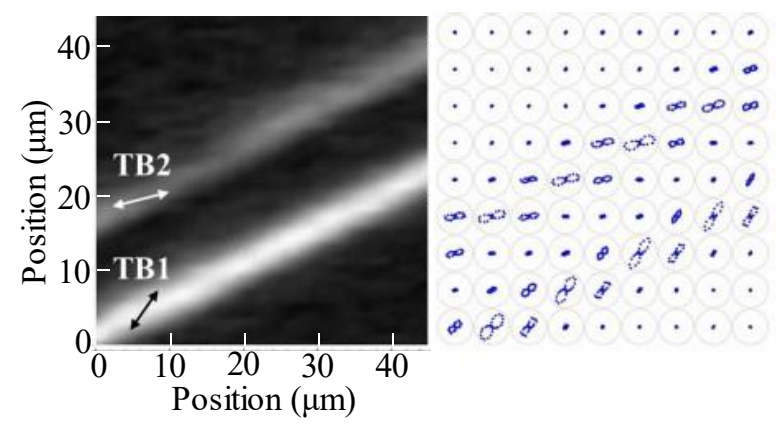

f Low energy electron microscopy After charge injection
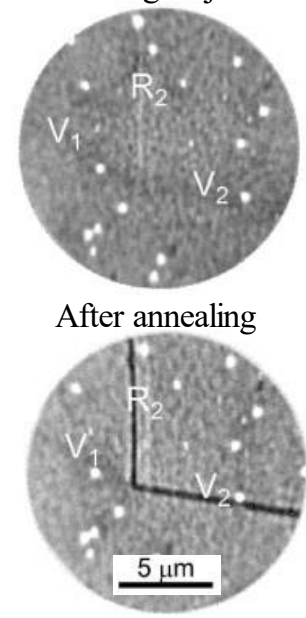

Fig. 3. Polar domain walls in non-polar $\mathrm{CaTiO}_{3}$. a $\mid$ Atomistic toy model of a domain pattern with multiple domain walls in view of the atomic shear strain $\varepsilon_{x y}$ (indicated by the colour code). b | Resulting dipole configuration (indicated by black arrows) along domain walls and vortices near domain wall intersections. Dipole displacements are amplified by a factor of 25. The junction density is high leading to extended vortex structures in $P$.

Reproduced with permission from REF. ${ }^{76}$. $\mathbf{c} \mid$ On the left, phase of the TEM reconstructed exit wave where the mean displacements of the Ti atomic columns from the centre of the four neighbouring $\mathrm{Ca}$ atomic columns are indicated by green arrows. On the right, displacements of $\mathrm{Ti}$ atomic columns in the y-directions averaged along and in mirror operation with respect to the twin wall together with their $90 \%$ confidence intervals. Adapted with permission from REF. ${ }^{81}$. d | Resonance piezoelectric spectra of a single crystal $\mathrm{CaTiO}_{3}$ sample. An AC electric field of $50 \mathrm{~V} \mathrm{~mm}^{-1}$ was applied to obtain the spectra. The spectra are vertically translated so that the axis is labelled as temperature. Adapted with permission from REF. ${ }^{33}$. e $\mid$ SHG image of an area that contains two SHG active domain walls: TB1 and TB2. The corresponding polar diagrams mapping is shown in the right panel. Adapted with permission from REF. ${ }^{71}$. f | Low energy electron microscopy images containing three domain walls $\left(\mathrm{V}_{1}, \mathrm{~V}_{2}, \mathrm{R}_{2}\right)$ after 10min exposure to $20 \mathrm{~V}$ e-beam, and the same regions after annealing at $330^{\circ} \mathrm{C}$. Adapted with permission from REF. ${ }^{33}$. 


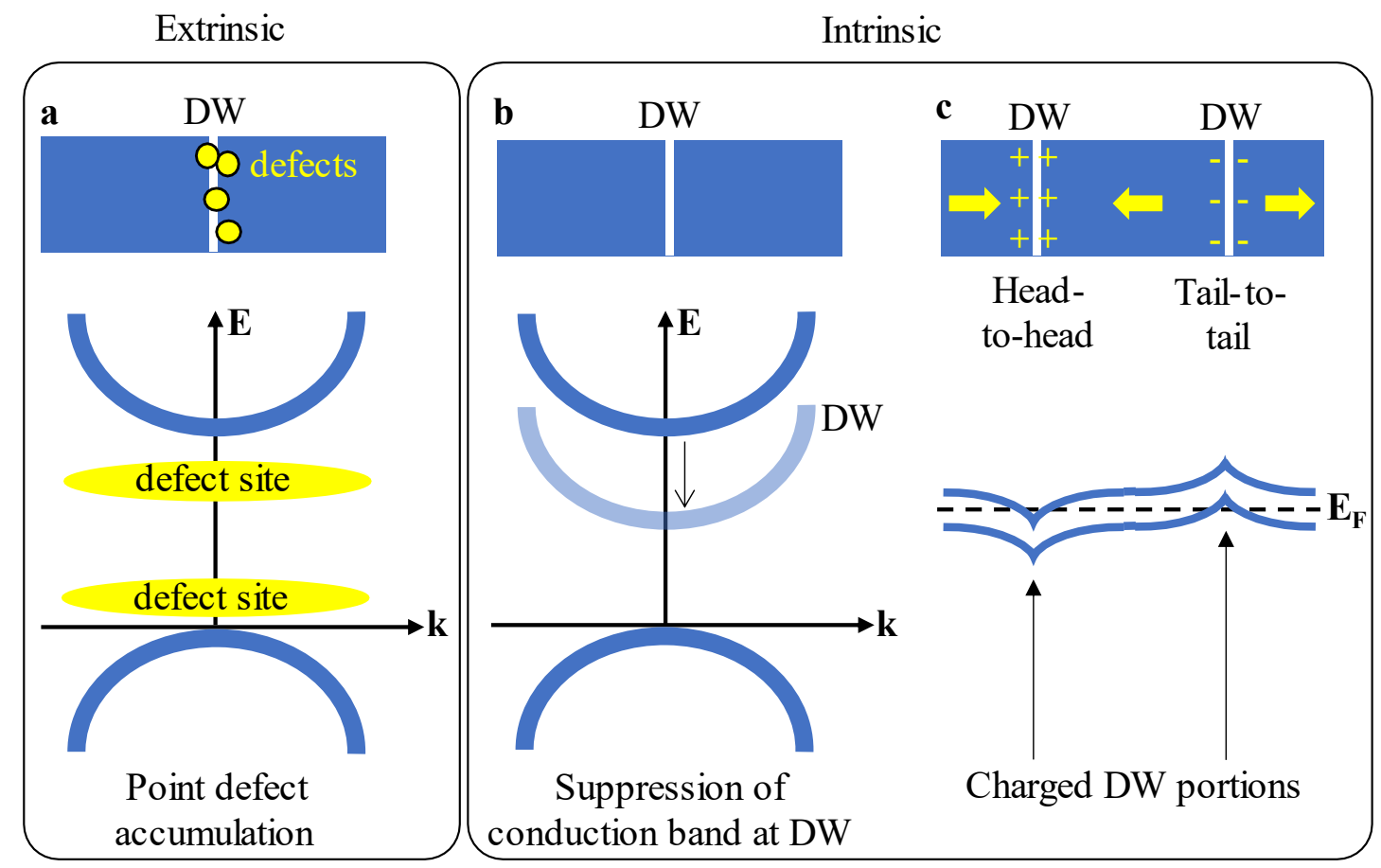

Fig. 4. Typical mechanisms of domain wall (DW) conduction. a | Intra-bandgap defect states between the conduction band (top semi-circle) and the valence band (bottom semicircle). Usually, intra-bandgap states are caused by defects but it may also be intrinsic to the pristine domain wall ${ }^{105}$. $\mathbf{b} \mid$ Intrinsic different electronic band structure of the domain wall leading to a reduction in the bandgap. $\mathbf{c} \mid$ Shift of the band structure induced by local electric fields associated with local head-to-head (giving positive bound charges "+") and tail-to-tail (giving negative bound charges "-") configurations of the polarisation (indicated by yellow arrows). $E$ and $k$ indicate the energy and the wave vector, respectively. $E_{\mathrm{F}}$ is the Fermi energy. 

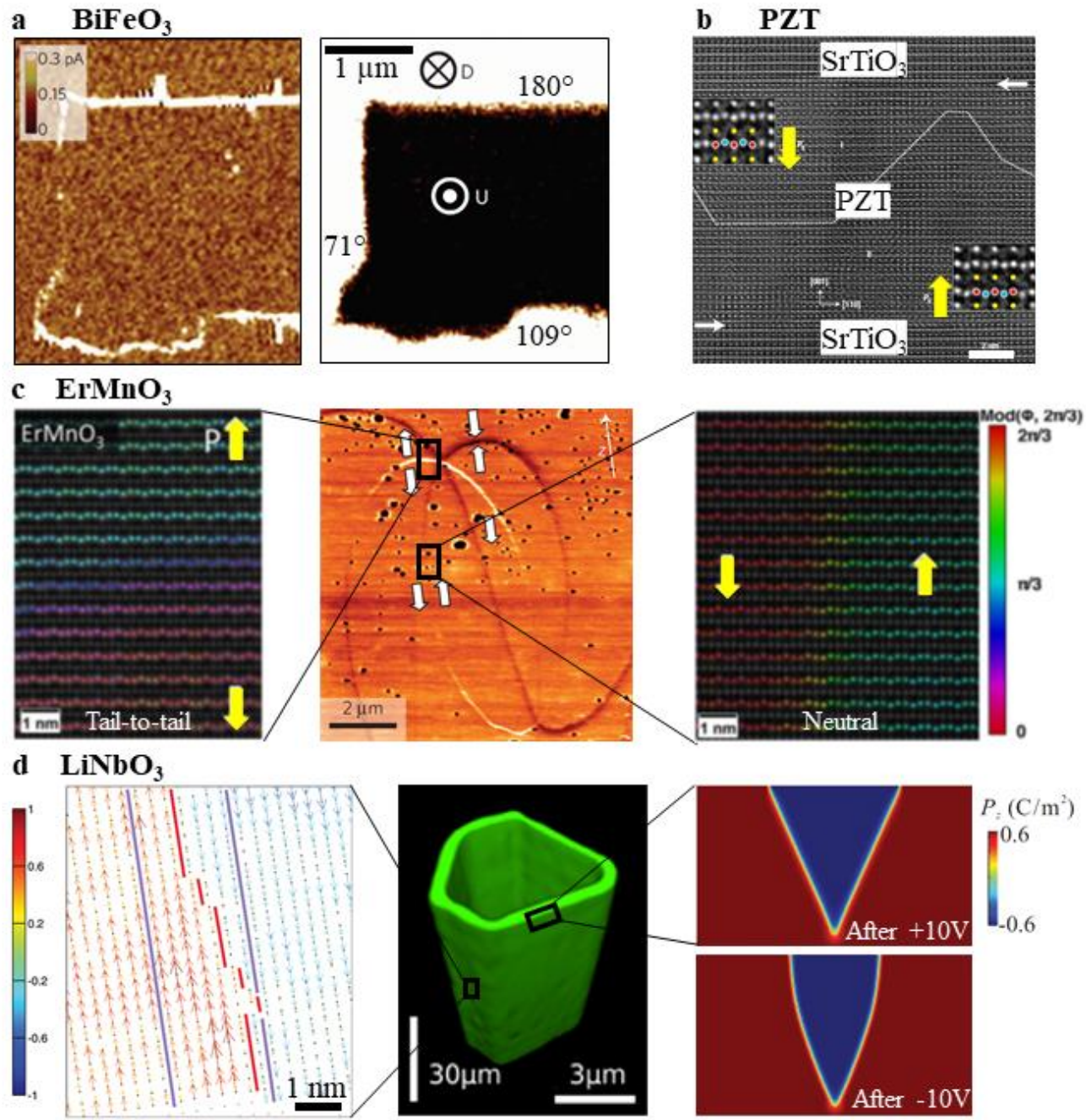

Fig. 5. Conduction at $180^{\circ}$ domain walls. a $\mid c-A F M$ (left) and out-of-plane piezoresponse force microscopy (right) images of a written domain pattern in a monodomain $\mathrm{BiFeO}_{3}$ film showing the out-of-plane polarisation component of the domains to be either down, labelled as ' $\mathrm{D}$ ' (white), or up, labelled as ' $U$ ' (black), with conduction at $109^{\circ}$ and $180^{\circ}$ domain walls, but not at $71^{\circ}$. Adapted with permission from REF. ${ }^{94} \cdot \mathbf{b} \mid$ TEM image of a

$\mathrm{SrTiO}_{3} / \mathrm{PbZr}_{0.2} \mathrm{Ti}_{0.8} \mathrm{O}_{3} / \mathrm{SrTiO}_{3}$ thin film heterostructure. The image is recorded under negative spherical-aberration imaging conditions with the incident electron beam parallel to the [-110] direction. The atom columns appear bright on a dark background. The horizontal arrows denote the horizontal interfaces between the PZT and the top and the bottom $\mathrm{SrTiO}_{3}$ film layers. The dotted line traces the $180^{\circ}$ domain wall. The yellow arrows show the directions of the polarisation in the $180^{\circ}$ domains. The insets show magnifications of the dipoles formed by the displacements of ions in the unit cells (yellow: $\mathrm{PbO}$, red: $\mathrm{Zr} / \mathrm{Ti}$, blue: $\mathrm{O}$ ). The white scalebar corresponds to $2 \mathrm{~nm}$. Adapted with permission from REF. ${ }^{7}$. $\mid$ |c-AFM image of two neighbouring singularities in the $y z$-plane of $\mathrm{ErMnO}_{3}$ with polarisation directions in-plane as indicated by the arrows. The inset on the left shows high-angle annular dark-field imagingSTEM (HAADF-STEM) images of negatively charged tail-to-tail domain walls and the inset on the right HAADF-STEM images of a neutral domain wall. The colour overlay of the phase describes the Er displacement. Adapted with permission from REF. ${ }^{113,115}$. d $\mid$ Threedimensional profile of an inclined domain wall in $\mathrm{LiNbO}_{3}$ by Cherenkov second-harmonic generation (centre). The inset on the left shows the analysis of 25 atomic rows from a STEM image: grey dots correspond to the fitted atom column positions, red and blue arrows indicate the displacement of the fitted atomic positions with respect to the base line and pointing in the direction of the displacement (the larger the displacement, the darker and longer the arrow). The inset on the right is a phase-field modelling of the domain wall profile after the application of $+/-10 \mathrm{~V}$. Bending of the upper segments of wall close to the surface can be clearly seen. Adapted with permission from REF., ${ }^{6,129,131}$. 


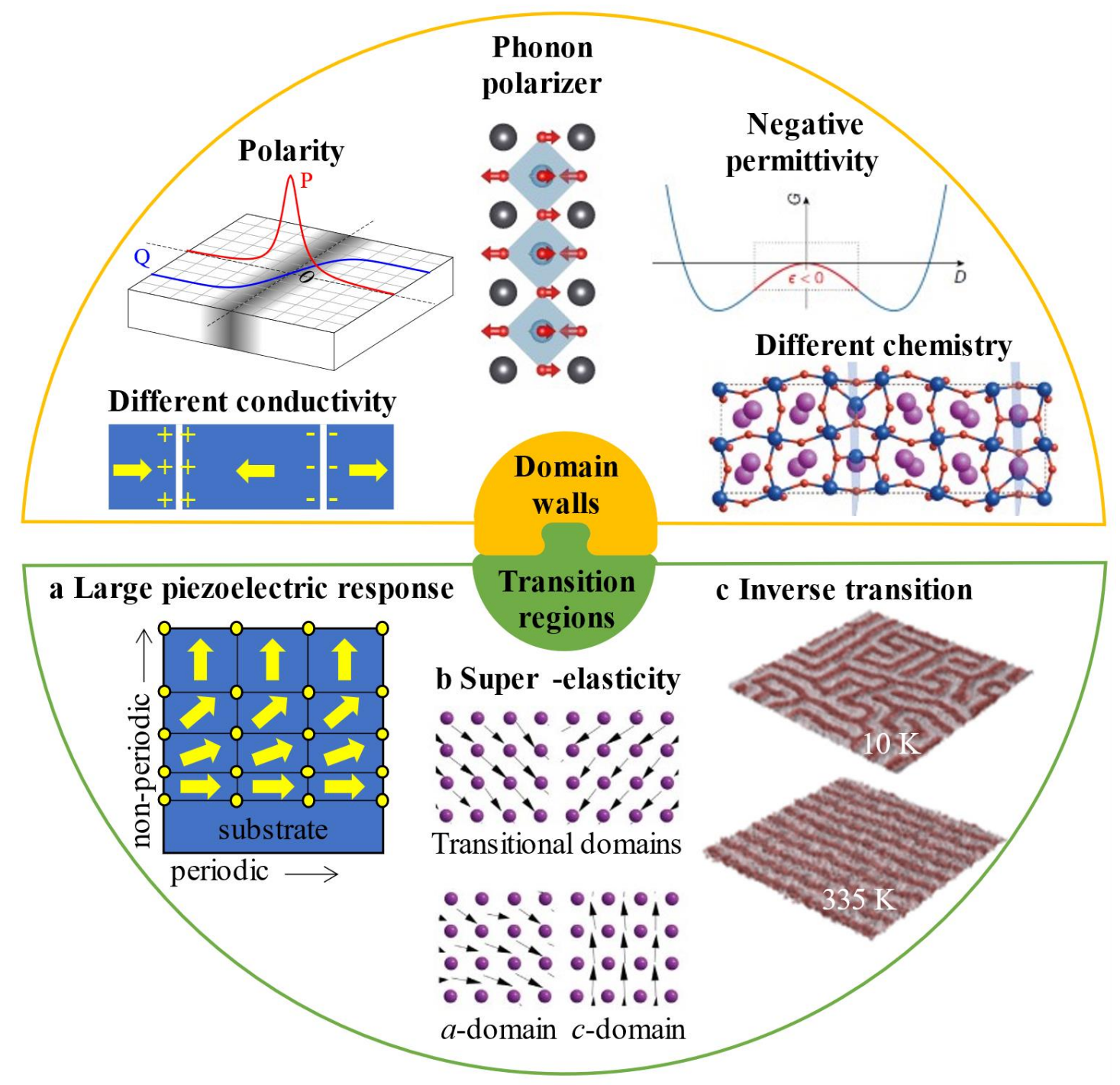

Fig. 6. Properties of domain walls and transition regions. Schematic view of the properties of domain walls (top semi-circle) and transitions regions (a-c). The images for domain walls are adapted from literature: phonon polarizer ${ }^{161}$, negative permittivity ${ }^{156}$, different chemistry ${ }^{162}$. a $\mid$ Schematic of a transitional ferroelectric with a gradual change in symmetry. Adapted with permission from REF. ${ }^{229}$. b|Dipole configurations in four selected areas of a bent membrane by atomistic simulations, featuring $a$-domains, $c$-domains and transitional domains. Adapted with permission from REF. ${ }^{177}$. $\mathbf{c |}$ Evolution of the labyrinthine pattern $(10 \mathrm{~K})$ to a stripe pattern $(335 \mathrm{~K})$ with increasing temperature. Adapted with permission from REF. ${ }^{178}$. 


\section{References}

1. Aizu, K. Possible Species of Ferromagnetic, Ferroelectric, and Ferroelastic Crystals. Phys. Rev. B 2, 754-772 (1970).

2. Wadhawan, V. Introduction to Ferroic Materials. (Gordon and Breach Science Publishers, 2000).

3. Van Aken, B. B., Rivera, J.-P., Schmid, H. \& Fiebig, M. Observation of ferrotoroidic domains. Nature 449, 702-705 (2007).

4. Schmid, H. Multi-ferroic magnetoelectrics. Ferroelectrics 162, 317-338 (1994).

5. Fiebig, M., Lottermoser, T., Meier, D. \& Trassin, M. The evolution of multiferroics. Nat. Rev. Mater. 1, 16046 (2016).

6. Gonnissen, J. et al. Direct Observation of Ferroelectric Domain Walls in $\mathrm{LiNbO}_{3}$ : Wall-Meanders, Kinks, and Local Electric Charges. Adv. Funct. Mater. 26, 7599-7604 (2016).

7. Jia, C.-L. et al. Atomic-scale study of electric dipoles near charged and uncharged domain walls in ferroelectric films. Nat. Mater. 7, 57-61 (2008).

8. Jia, C.-L., Urban, K. W., Alexe, M., Hesse, D. \& Vrejoiu, I. Direct Observation of Continuous Electric Dipole Rotation in Flux-Closure Domains in Ferroelectric $\mathrm{Pb}(\mathrm{Zr}, \mathrm{Ti}) \mathrm{O}_{3}$. Science 331, 1420-1423 (2011).

9. Salje, E. K. H. \& Scott, J. F. Ferroelectric Bloch-line switching: A paradigm for memory devices? Appl. Phys. Lett. 105, 252904 (2014).

10. Stepkova, V., Marton, P. \& Hlinka, J. Ising lines: Natural topological defects within ferroelectric Bloch walls. Phys. Rev. B 92, 094106 (2015).

11. Seidel, J. (editor). Topological Structures in Ferroic Materials. (Springer International Publishing, 2016).

12. Stepkova, V. \& Hlinka, J. On the possible internal structure of the ferroelectric Ising lines in $\mathrm{BaTiO}_{3}$. Phase Transitions 90, 11-16 (2017).

13. Parkin, S. S. P., Hayashi, M. \& Thomas, L. Magnetic Domain-Wall Racetrack Memory. Science 320, 190-194 (2008).

14. Al Bahri, M. et al. Staggered Magnetic Nanowire Devices for Effective Domain-Wall Pinning in Racetrack Memory. Phys. Rev. Appl. 11, 024023 (2019).

15. Harrison, R. J., Redfern, S. A. T., Buckley, A. \& Salje, E. K. H. Application of real-time, stroboscopic X-ray diffraction with dynamical mechanical analysis to characterize the motion of ferroelastic domain walls. J. Appl. Phys. 95, 17061717 (2004).

16. Schilling, A. et al. Scaling of domain periodicity with thickness measured in $\mathrm{BaTiO}_{3}$ single crystal lamellae and comparison with other ferroics. Phys. Rev. B 74, 024115 (2006).

17. Yang, S. Y. et al. Above-bandgap voltages from ferroelectric photovoltaic devices. Nat. Nanotechnol. 5, 143-147 (2010).

18. Vul, B. M., Guro, G. M. \& Ivanchik, I. I. Encountering domains in ferroelectrics. Ferroelectrics 6, 29-31 (1973).

19. Catalan, G., Seidel, J., Ramesh, R. \& Scott, J. F. Domain wall nanoelectronics. Rev. Mod. Phys. 84, 119-156 (2012).

20. Sharma, P., Schoenherr, P. \& Seidel, J. Functional Ferroic Domain Walls for Nanoelectronics. Materials 12, 2927 (2019).

21. Seidel, J. Domain Walls as Nanoscale Functional Elements. J. Phys. Chem. Lett. 3, 2905-2909 (2012).

22. Salje, E. K. H. Multiferroic Domain Boundaries as Active Memory Devices: 
Trajectories Towards Domain Boundary Engineering. ChemPhysChem 11, 940-950 (2010).

23. Whyte, J. R. \& Gregg, J. M. A diode for ferroelectric domain-wall motion. Nat. Commun. 6, 7361 (2015).

24. Sharma, P. et al. Nonvolatile ferroelectric domain wall memory. Sci. Adv. 3, 19 (2017).

25. Sharma, P. et al. Conformational Domain Wall Switch. Adv. Funct. Mater. 29, 1807523 (2019).

26. Jiang, J. et al. Temporary formation of highly conducting domain walls for non-destructive read-out of ferroelectric domain-wall resistance switching memories. Nat. Mater. 17, 49-55 (2018).

27. McConville, J. P. V. et al. Ferroelectric Domain Wall Memristor. Adv. Funct. Mater. 2000109 (2020).

28. Chai, X. et al. Nonvolatile ferroelectric field-effect transistors. Nat. Commun. 11, 2811 (2020).

29. Bai, Z. L. et al. Hierarchical Domain Structure and Extremely Large Wall Current in Epitaxial $\mathrm{BiFeO}_{3}$ Thin Films. Adv. Funct. Mater. 28, 1801725 (2018).

30. Sanchez-Santolino, G. et al. Resonant electron tunnelling assisted by charged domain walls in multiferroic tunnel junctions. Nat. Nanotechnol. 12, 655-662 (2017).

31. Mundy, J. A. et al. Functional electronic inversion layers at ferroelectric domain walls. Nat. Mater. 16, 622-627 (2017).

32. Schaab, J. et al. Electrical half-wave rectification at ferroelectric domain walls. Nat. Nanotechnol. 13, 1028-1034 (2018).

33. Nataf, G. F. et al. Control of surface potential at polar domain walls in a nonpolar oxide. Phys. Rev. Mater. 1, 074410 (2017).

34. Frenkel, Y. et al. Imaging and tuning polarity at $\mathrm{SrTiO}_{3}$ domain walls. Nat. Mater. 16, 1203-1208 (2017).

35. Bednyakov, P. S., Sturman, B. I., Sluka, T., Tagantsev, A. K. \& Yudin, P. V. Physics and applications of charged domain walls. npj Comput. Mater. 4, 65 (2018).

36. Seidel, J., Vasudevan, R. K. \& Valanoor, N. Topological Structures in Multiferroics - Domain Walls, Skyrmions and Vortices. Adv. Electron. Mater. 2, 1500292 (2016).

37. Seidel, J. Nanoelectronics based on topological structures. Nat. Mater. 18, 188 190 (2019).

38. Meier, D. Functional domain walls in multiferroics. J. Phys. Condens. Matter 27, 463003 (2015).

39. Tagantsev, A. K., Cross, L. E. \& Fousek, J. Domains in Ferroic Crystals and Thin Films. (Springer, 2010).

40. Zhirnov, V. A. A Contribution To the Theory of Domain Walls in Ferroelectrics. Sov. Phys. JETP 35, 822-825 (1959).

41. Lawless, W. N. \& Fousek, J. Small-Signal Permittivity of the Stationary (100)$180^{\circ}$ Domain Wall in $\mathrm{BaTiO}_{3}$. J. Phys. Soc. Japan 28, 419-424 (1970).

42. Lajzerowicz, J. \& Niez, J. J. Phase transition in a domain wall. J. Phys. Lettres 40, 165-169 (1979).

43. Houchmandzadeh, B., Lajzerowicz, J. \& Salje, E. Order parameter coupling and chirality of domain walls. J. Phys. Condens. Matter 3, 5163-5169 (1991).

44. Bul'bich, A. A. \& Gufan, Y. M. Inevitable symmetry lowering in a domain wall near a reordering phase transition. Zh. Eksp. Teor. Fiz. 94, 121 (1988). 
45. Marton, P., Rychetsky, I. \& Hlinka, J. Domain walls of ferroelectric $\mathrm{BaTiO}_{3}$ within the Ginzburg-Landau-Devonshire phenomenological model. Phys. Rev. $B$ 81, 144125 (2010).

46. Hlinka, J. et al. Phase-field modelling of $180^{\circ}$ "Bloch walls" in rhombohedral $\mathrm{BaTiO}_{3}$. Phase Transitions 84, 738-746 (2011).

47. Stepkova, V., Marton, P. \& Hlinka, J. Stress-induced phase transition in ferroelectric domain walls of $\mathrm{BaTiO}_{3}$. J. Phys. Condens. Matter 24, 212201 (2012).

48. Marton, P., Stepkova, V. \& Hlinka, J. Divergence of dielectric permittivity near phase transition within ferroelectric domain boundaries. Phase Transitions 86, 103-108 (2013).

49. Wojdeł, J. C. \& Íñiguez, J. Ferroelectric Transitions at Ferroelectric Domain Walls Found from First Principles. Phys. Rev. Lett. 112, 247603 (2014).

50. Gu, Y. et al. Flexoelectricity and ferroelectric domain wall structures: Phasefield modeling and DFT calculations. Phys. Rev. B 89, 174111 (2014).

51. Morozovska, A. N., Kalinin, S. V.\& Eliseev, E. A. Flexoelectricity Impact on the Domain Wall Structure and Polar Properties. in Flexoelectricity in Solids 311-336 (World Scientific, 2016).

52. Lee, D. et al. Mixed Bloch-Néel-Ising character of $180^{\circ}$ ferroelectric domain walls. Phys. Rev. B 80, 060102 (2009).

53. Taherinejad, M., Vanderbilt, D., Marton, P., Stepkova, V. \& Hlinka, J. Blochtype domain walls in rhombohedral $\mathrm{BaTiO}_{3}$. Phys. Rev. B 86, 155138 (2012).

54. Yudin, P. V., Tagantsev, a. K. \& Setter, N. Bistability of ferroelectric domain walls: Morphotropic boundary and strain effects. Phys. Rev. B 88, 024102 (2013).

55. Cherifi-Hertel, S. et al. Non-Ising and chiral ferroelectric domain walls revealed by nonlinear optical microscopy. Nat. Commun. 8, 15768 (2017).

56. Wei, X.-K. et al. Néel-like domain walls in ferroelectric $\mathrm{Pb}(\mathrm{Zr}, \mathrm{Ti}) \mathrm{O}_{3}$ single crystals. Nat. Commun. 7, 12385 (2016).

57. De Luca, G. et al. Domain Wall Architecture in Tetragonal Ferroelectric Thin Films. Adv. Mater. 29, 1-5 (2017).

58. Janovec, V. A symmetry approach to domain structures. Ferroelectrics 12, 4353 (1976).

59. Janovec, V. Symmetry and structure of domain walls. Ferroelectrics 35, 105110 (1981).

60. Janovec, V. \& Př́vratská, J. Domain structures. in International Tables for Crystallography 449-505 (International Union of Crystallography, 2006). doi:10.1107/97809553602060000645

61. Janovec, V.\& Kopský, V. Layer groups, scanning tables and the structure of domain walls. Ferroelectrics 191, 23-28 (1997).

62. Janovec, V., Schranz, W., Warhanek, H. \& Zikmund, Z. Symmetry analysis of domain structure in KSCN crystals. Ferroelectrics 98, 171-189 (1989).

63. Kopský, V. The Scanning for Layer Groups and Positional Dependence of Domain Wall Energy and Structure. Ferroelectrics 376, 168-175 (2008).

64. Janovec, V., Grocký, M., Kopský, V. \& Kluiber, Z. On Atomic Displacements in $90^{\circ}$ Ferroelectric Domain Walls of Tetragonal $\mathrm{BaTiO}_{3}$ Crystals.

Ferroelectrics 303, 65-68 (2004).

65. Janovec, V. \& Litvin, D. B. Symmetry-allowed atomic displacements in a ferroelastic domain wall of rhombohedral $\mathrm{BaTiO}_{3}$. Phase Transitions $\mathbf{8 4}, 760$ 768 (2011).

66. Přívratská, J. \& Janovec, V. Examination of point group symmetries of non- 
ferroelastic domain walls. Ferroelectrics 191, 17-21 (1997).

67. Přívratská, J., Janovec, V. \& Machonský, L. Tensor properties discriminating domain walls from non-ferroelastic domains. Ferroelectrics 240, 1349-1358 (2000).

68. Tolédano, P., Guennou, M. \& Kreisel, J. Order-parameter symmetries of domain walls in ferroelectrics and ferroelastics. Phys. Rev. B 89, 134104 (2014).

69. Schranz, W., Rychetsky, I. \& Hlinka, J. Polarity of domain boundaries in nonpolar materials derived from order parameter and layer group symmetry. Phys. Rev. B 100, 184105 (2019).

70. Janovec, V., Richterová, L. \& Př́vratská, J. Polar properties of compatible ferroelastic domain walls. Ferroelectrics 222, 73-76 (1999).

71. Yokota, H. et al. Direct evidence of polar nature of ferroelastic twin boundaries in $\mathrm{CaTiO}_{3}$ obtained by second harmonic generation microscope. Phys. Rev. B 89, 144109 (2014).

72. Yokota, H., Matsumoto, S., Salje, E. K. H. \& Uesu, Y. Symmetry and threedimensional anisotropy of polar domain boundaries observed in ferroelastic $\mathrm{LaAlO}_{3}$ in the complete absence of ferroelectric instability. Phys. Rev. B 98, 104105 (2018).

73. Yokota, H., Matsumoto, S., Salje, E. K. H. \& Uesu, Y. Polar nature of domain boundaries in purely ferroelastic $\mathrm{Pb}_{3}\left(\mathrm{PO}_{4}\right)_{2}$ investigated by second harmonic generation microscopy. Phys. Rev. B 100, 024101 (2019).

74. Yokota, H., Matsumoto, S., Hasegawa, N., Salje, E. \& Uesu, Y. Enhancement of polar nature of domain boundaries in ferroelastic $\mathrm{Pb}_{3}\left(\mathrm{PO}_{4}\right)_{2}$ by doping divalent-metal ions. J. Phys. Condens. Matter 32, 345401 (2020).

75. Yokota, H., Hasegawa, N., Glazer, M., Salje, E. K. H. \& Uesu, Y. Direct evidence of polar ferroelastic domain boundaries in semiconductor $\mathrm{BiVO}_{4}$. Appl. Phys. Lett. 116, 232901 (2020).

76. Salje, E. K. H., Li, S., Stengel, M., Gumbsch, P. \& Ding, X. Flexoelectricity and the polarity of complex ferroelastic twin patterns. Phys. Rev. B 94, 024114 (2016).

77. Goncalves-Ferreira, L., Redfern, S. A. T., Artacho, E. \& Salje, E. K. H. Ferrielectric Twin Walls in $\mathrm{CaTiO}_{3}$. Phys. Rev. Lett. 101, 097602 (2008).

78. Conti, S., Müller, S., Poliakovsky, A. \& Salje, E. K. H. Coupling of order parameters, chirality, and interfacial structures in multiferroic materials. $J$. Phys. Condens. Matter 23, 142203 (2011).

79. Pöttker, H. \& Salje, E. K. H. Flexoelectricity, incommensurate phases and the Lifshitz point. J. Phys. Condens. Matter 28, 075902 (2016).

80. Pöttker, H. \& Salje, E. K. H. Twin boundary profiles with linear-quadratic coupling between order parameters. J. Phys. Condens. Matter 26, 342201 (2014).

81. Van Aert, S. et al. Direct Observation of Ferrielectricity at Ferroelastic Domain Boundaries in $\mathrm{CaTiO}_{3}$ by Electron Microscopy. Adv. Mater. 24, 523-527 (2012).

82. Salje, E. K. H., Aktas, O., Carpenter, M. A., Laguta, V. V. \& Scott, J. F. Domains within Domains and Walls within Walls: Evidence for Polar Domains in Cryogenic SrTiO 3 . Phys. Rev. Lett. 111, 247603 (2013).

83. Zhao, Z. et al. Interaction of low-energy electrons with surface polarity near ferroelastic domain boundaries. Phys. Rev. Mater. 3, 043601 (2019).

84. Casals, B. et al. Low-Temperature Dielectric Anisotropy Driven by an Antiferroelectric Mode in $\mathrm{SrTiO}_{3}$. Phys. Rev. Lett. 120, 217601 (2018). 
85. Pesquera, D., Carpenter, M. A. \& Salje, E. K. H. Glasslike Dynamics of Polar Domain Walls in Cryogenic $\mathrm{SrTiO}_{3}$. Phys. Rev. Lett. 121, 235701 (2018).

86. Novak, J. \& Salje, E. K. H. Surface structure of domain walls. J. Phys. Condens. Matter 10, L359-L366 (1998).

87. Nataf, G. F. et al. Low energy electron imaging of domains and domain walls in magnesium-doped lithium niobate. Sci. Rep. 6, 33098 (2016).

88. Barrett, N. et al. Full field electron spectromicroscopy applied to ferroelectric materials. J. Appl. Phys. 113, 187217 (2013).

89. Lu, G., Li, S., Ding, X. \& Salje, E. K. H. Piezoelectricity and electrostriction in ferroelastic materials with polar twin boundaries and domain junctions. Appl. Phys. Lett. 114, 202901 (2019).

90. Lu, G., Li, S., Ding, X., Sun, J. \& Salje, E. K. H. Ferroelectric switching in ferroelastic materials with rough surfaces. Sci. Rep. 9, 15834 (2019).

91. Schmid, H. \& Pétermann, L. A. Dielectric constant and electric resistivity of copper chlorine boracite, $\mathrm{Cu}_{3} \mathrm{~B}_{7} \mathrm{O}_{13} \mathrm{Cl}(\mathrm{Cu}-\mathrm{Cl}-\mathrm{B})$. Phys. Status Solidi 41, K147K150 (1977).

92. Aird, A. \& Salje, E. K. H. Sheet superconductivity in twin walls: experimental evidence of $\mathrm{WO}_{3-\mathrm{x}}$. J. Phys. Condens. Matter 10, L377-L380 (1998).

93. Kim, Y., Alexe, M. \& Salje, E. K. H. Nanoscale properties of thin twin walls and surface layers in piezoelectric $\mathrm{WO}_{3-\mathrm{x}}$. Appl. Phys. Lett. 96, 032904 (2010).

94. Seidel, J. et al. Conduction at domain walls in oxide multiferroics. Nat. Mater. 8, 229-34 (2009).

95. Farokhipoor, S. \& Noheda, B. Local conductivity and the role of vacancies around twin walls of (001)- $\mathrm{BiFeO}_{3}$ thin films. J. Appl. Phys. 112, 052003 (2012).

96. Farokhipoor, S. \& Noheda, B. Conduction through $71^{\circ}$ Domain Walls in $\mathrm{BiFeO}_{3}$ Thin Films. Phys. Rev. Lett. 107, 127601 (2011).

97. Chiu, Y.-P. et al. Atomic-scale evolution of local electronic structure across multiferroic domain walls. Adv. Mater. 23, 1530-4 (2011).

98. Lubk, A., Gemming, S. \& Spaldin, N. First-principles study of ferroelectric domain walls in multiferroic bismuth ferrite. Phys. Rev. B 80, 104110 (2009).

99. Diéguez, O., Aguado-Puente, P., Junquera, J. \& Íñiguez, J. Domain walls in a perovskite oxide with two primary structural order parameters: First-principles study of $\mathrm{BiFeO}_{3}$. Phys. Rev. B 87, 024102 (2013).

100. Seidel, J. et al. Domain Wall Conductivity in La-Doped $\mathrm{BiFeO}_{3}$. Phys. Rev. Lett. 105, 197603 (2010).

101. Campanini, M. et al. Imaging and quantification of charged domain walls in $\mathrm{BiFeO}_{3}$. Nanoscale 12, 9186-9193 (2020).

102. Maksymovych, P. et al. Dynamic Conductivity of Ferroelectric Domain Walls in $\mathrm{BiFeO}_{3}$. Nano Lett. 11, 1906-1912 (2011).

103. Li, L. et al. Atomic Scale Structure Changes Induced by Charged Domain Walls in Ferroelectric Materials. Nano Lett. 13, 5218-5223 (2013).

104. Vasudevan, R. K. et al. Domain Wall Geometry Controls Conduction in Ferroelectrics. Nano Lett. 12, 5524-5531 (2012).

105. Körbel, S., Hlinka, J. \& Sanvito, S. Electron trapping by neutral pristine ferroelectric domain walls in $\mathrm{BiFeO}_{3}$. Phys. Rev. B 98, 100104(R) (2018).

106. Rojac, T. et al. Domain-wall conduction in ferroelectric $\mathrm{BiFeO}_{3}$ controlled by accumulation of charged defects. Nat. Mater. 16, 322-327 (2017).

107. Lee, J. H. et al. Spintronic Functionality of $\mathrm{BiFeO}_{3}$ Domain Walls. Adv. Mater. 26, 7078-82 (2014).

108. Stolichnov, I. et al. Persistent conductive footprints of $109^{\circ}$ domain walls in 
bismuth ferrite films. Appl. Phys. Lett. 104, 132902 (2014).

109. Domingo, N., Farokhipoor, S., Santiso, J., Noheda, B. \& Catalan, G. Domain wall magnetoresistance in $\mathrm{BiFeO} 3$ thin films measured by scanning probe microscopy. J. Phys. Condens. Matter 29, 334003 (2017).

110. He, Q. et al. Magnetotransport at Domain Walls in $\mathrm{BiFeO}_{3}$. Phys. Rev. Lett. 108, 067203 (2012).

111. Yang, J. C. et al. Conduction control at ferroic domain walls via external stimuli. Nanoscale 6, 10524-10529 (2014).

112. Choi, T. et al. Insulating interlocked ferroelectric and structural antiphase domain walls in multiferroic $\mathrm{YMnO}_{3}$. Nat. Mater. 9, 253-8 (2010).

113. Meier, D. et al. Anisotropic conductance at improper ferroelectric domain walls. Nat. Mater. 11, 284-288 (2012).

114. Wu, W., Horibe, Y., Lee, N., Cheong, S.-W. \& Guest, J. R. Conduction of Topologically Protected Charged Ferroelectric Domain Walls. Phys. Rev. Lett. 108, 077203 (2012).

115. Holtz, M. E. et al. Topological Defects in Hexagonal Manganites: Inner Structure and Emergent Electrostatics. Nano Lett. 17, 5883-5890 (2017).

116. Småbråten, D. R. et al. Charged domain walls in improper ferroelectric hexagonal manganites and gallates. Phys. Rev. Mater. 2, 114405 (2018).

117. Schoenherr, P. et al. Observation of Uncompensated Bound Charges at Improper Ferroelectric Domain Walls. Nano Lett. 19, 1659-1664 (2019).

118. Turner, P. W. et al. Large Carrier Mobilities in $\mathrm{ErMnO}_{3}$ Conducting Domain Walls Revealed by Quantitative Hall-Effect Measurements. Nano Lett. 18, 6381-6386 (2018).

119. Mosberg, A. B. et al. FIB lift-out of conducting ferroelectric domain walls in hexagonal manganites. Appl. Phys. Lett. 115, 122901 (2019).

120. Kumagai, Y. \& Spaldin, N. A. Structural domain walls in polar hexagonal manganites. Nat. Commun. 4, 1540 (2013).

121. Du, Y. et al. Domain wall conductivity in oxygen deficient multiferroic $\mathrm{YMnO}_{3}$ single crystals. Appl. Phys. Lett. 99, 252107 (2011).

122. Wu, X. et al. Low-energy structural dynamics of ferroelectric domain walls in hexagonal rare-earth manganites. Sci. Adv. 3, e1602371 (2017).

123. Sluka, T., Tagantsev, A. K., Bednyakov, P. \& Setter, N. Free-electron gas at charged domain walls in insulating $\mathrm{BaTiO}_{3}$. Nat. Commun. 4, 1808 (2013).

124. Gureev, M. Y., Tagantsev, A. K. \& Setter, N. Head-to-head and tail-to-tail $180^{\circ}$ domain walls in an isolated ferroelectric. Phys. Rev. B 83, 184104 (2011).

125. Sluka, T., Tagantsev, A. K., Damjanovic, D., Gureev, M. \& Setter, N. Enhanced electromechanical response of ferroelectrics due to charged domain walls. Nat. Commun. 3, 748 (2012).

126. Bednyakov, P. S., Sluka, T., Tagantsev, A. K., Damjanovic, D. \& Setter, N. Formation of charged ferroelectric domain walls with controlled periodicity. Sci. Rep. 5, 15819 (2015).

127. Aristov, V. V., Kokhanchik, L. S. \& Voronovskii, Y. I. Voltage contrast of ferroelectric domains of lithium niobate in SEM. Phys. Status Solidi 86, 133141 (1984).

128. Schröder, M. et al. Conducting Domain Walls in Lithium Niobate Single Crystals. Adv. Funct. Mater. 22, 3936-3944 (2012).

129. Kämpfe, T. et al. Optical three-dimensional profiling of charged domain walls in ferroelectrics by Cherenkov second-harmonic generation. Phys. Rev. B 89, 035314 (2014).

130. Sheng, Y. et al. Three-dimensional ferroelectric domain visualization by 
Čerenkov-type second harmonic generation. Opt. Express 18, 16539 (2010).

131. Lu, H. et al. Electrical Tunability of Domain Wall Conductivity in $\mathrm{LiNbO}_{3}$ Thin Films. Adv. Mater. 31, 1902890 (2019).

132. Pryakhina, V. I. et al. As-grown domain structure in lithium tantalate with spatially nonuniform composition. Ferroelectrics 525, 47-53 (2018).

133. Greshnyakov, E. D., Lisjikh, B. I., Pryakhina, V. I., Nebogatikov, M. S. \& Shur, V. Y. Charged domain walls in lithium tantalate with compositional gradients produced by partial VTE process. IOP Conf. Ser. Mater. Sci. Eng. 699, 012015 (2019).

134. Eliseev, E. A., Morozovska, A. N., Svechnikov, G. S., Gopalan, V. \& Shur, V. Y. Static conductivity of charged domain walls in uniaxial ferroelectric semiconductors. Phys. Rev. B 83, 235313 (2011).

135. Godau, C., Kämpfe, T., Thiessen, A., Eng, L. M. \& Haußmann, A. Enhancing the Domain Wall Conductivity in Lithium Niobate Single Crystals. ACS Nano 11, 4816-4824 (2017).

136. Schröder, M. et al. Nanoscale and macroscopic electrical ac transport along conductive domain walls in lithium niobate single crystals. Mater. Res. Express 1, 035012 (2014).

137. Werner, C. S. et al. Large and accessible conductivity of charged domain walls in lithium niobate. Sci. Rep. 7, 9862 (2017).

138. Nataf, G. F., Guennou, M., Haußmann, A., Barrett, N. \& Kreisel, J. Evolution of defect signatures at ferroelectric domain walls in $\mathrm{Mg}$-doped $\mathrm{LiNbO}_{3}$. Phys. status solidi - Rapid Res. Lett. 10, 222-226 (2016).

139. Nataf, G. F., Aktas, O., Granzow, T. \& Salje, E. K. H. Influence of defects and domain walls on dielectric and mechanical resonances in $\mathrm{LiNbO}_{3}$. J. Phys. Condens. Matter 28, 015901 (2016).

140. Wu, X.\& Vanderbilt, D. Theory of hypothetical ferroelectric superlattices incorporating head-to-head and tail-to-tail $180^{\circ}$ domain walls. Phys. Rev. B 73, 020103 (2006).

141. Rahmanizadeh, K., Wortmann, D., Bihlmayer, G. \& Blügel, S. Charge and orbital order at head-to-head domain walls in $\mathrm{PbTiO}_{3}$. Phys. Rev. B 90, 115104 (2014).

142. Guyonnet, J., Gaponenko, I., Gariglio, S. \& Paruch, P. Conduction at domain walls in insulating $\mathrm{Pb}\left(\mathrm{Zr}_{0.2} \mathrm{Ti}_{0.8}\right) \mathrm{O}_{3}$ thin films. Adv. Mater. 23, 5377-82 (2011).

143. Eliseev, E. A., Morozovska, A. N., Svechnikov, G. S., Maksymovych, P. \& Kalinin, S. V. Domain wall conduction in multiaxial ferroelectrics. Phys. Rev. B 85, 045312 (2012).

144. Sifuna, J., García-Fernández, P., Manyali, G. S., Amolo, G. \& Junquera, J. First-principles study of two-dimensional electron and hole gases at the headto-head and tail-to-tail $180^{\circ}$ domain walls in $\mathrm{PbTiO}_{3}$ ferroelectric thin films. Phys. Rev. B 101, 174114 (2020).

145. Gaponenko, I., Tückmantel, P., Karthik, J., Martin, L. W. \& Paruch, P. Towards reversible control of domain wall conduction in $\mathrm{Pb}\left(\mathrm{Zr}_{0.2} \mathrm{Ti}_{0.8}\right) \mathrm{O}_{3}$ thin films. Appl. Phys. Lett. 106, 162902 (2015).

146. Tselev, A. et al. Microwave a.c. conductivity of domain walls in ferroelectric thin films. Nat. Commun. 7, 11630 (2016).

147. Maksymovych, P. et al. Tunable metallic conductance in ferroelectric nanodomains. Nano Lett. 12, 209-213 (2012).

148. Stolichnov, I. et al. Bent Ferroelectric Domain Walls as Reconfigurable Metallic-Like Channels. Nano Lett. 15, 8049-8055 (2015).

149. Wei, X.-K. et al. Controlled Charging of Ferroelastic Domain Walls in Oxide 
Ferroelectrics. ACS Appl. Mater. Interfaces 9, 6539-6546 (2017).

150. Seidel, J. et al. Efficient Photovoltaic Current Generation at Ferroelectric Domain Walls. Phys. Rev. Lett. 107, 126805 (2011).

151. Seidel, J., Yang, S. Y., Alarcón-Lladó, E., Ager, J. W. \& Ramesh, R. Nanoscale probing of high photovoltages at $109^{\circ}$ domain walls. Ferroelectrics 433, 123-126 (2012).

152. Bhatnagar, A., Roy Chaudhuri, A., Heon Kim, Y., Hesse, D. \& Alexe, M. Role of domain walls in the abnormal photovoltaic effect in $\mathrm{BiFeO}_{3}$. Nat. Commun. 4, 2835 (2013).

153. Yang, M.-M., Bhatnagar, A., Luo, Z.-D. \& Alexe, M. Enhancement of Local Photovoltaic Current at Ferroelectric Domain Walls in $\mathrm{BiFeO}_{3}$. Sci. Rep. 7, 43070 (2017).

154. Nataf, G. F. \& Guennou, M. Optical studies of ferroelectric and ferroelastic domain walls. J. Phys. Condens. Matter 32, 183001 (2020).

155. Balcells, L. et al. Enhanced conduction and ferromagnetic order at (100)-type twin walls in $\mathrm{La}_{0.7} \mathrm{Sr}_{0.3} \mathrm{MnO}_{3}$ thin films. Phys. Rev. B 92, 075111 (2015).

156. Yadav, A. K. et al. Spatially resolved steady-state negative capacitance. Nature 565, 468-471 (2019).

157. Zubko, P. et al. Negative capacitance in multidomain ferroelectric superlattices. Nature 534, 524-528 (2016).

158. Islam Khan, A. et al. Experimental evidence of ferroelectric negative capacitance in nanoscale heterostructures. Appl. Phys. Lett. 99, 113501 (2011).

159. Salahuddin, S. \& Datta, S. Use of Negative Capacitance to Provide Voltage Amplification for Low Power Nanoscale Devices. Nano Lett. 8, 405-410 (2008).

160. Stefani, C. et al. Ferroelectric 180 degree walls are mechanically softer than the domains they separate. (2020). arxiv ID: 2005.04249

161. Royo, M., Escorihuela-Sayalero, C., Íñiguez, J. \& Rurali, R. Ferroelectric domain wall phonon polarizer. Phys. Rev. Mater. 1, 051402 (2017).

162. Farokhipoor, S. et al. Artificial chemical and magnetic structure at the domain walls of an epitaxial oxide. Nature 515, 379-383 (2014).

163. Bibes, M. \& Barthelemy, A. Oxide Spintronics. IEEE Trans. Electron Devices 54, 1003-1023 (2007).

164. Kruglyak, V. V., Demokritov, S. O. \& Grundler, D. Magnonics. J. Phys. D. Appl. Phys. 43, 264001 (2010).

165. Becher, C. et al. Strain-induced coupling of electrical polarization and structural defects in $\mathrm{SrMnO}_{3}$ films. Nat. Nanotechnol. 10, 661-665 (2015).

166. Becher, C. et al. Functional ferroic heterostructures with tunable integral symmetry. Nat. Commun. 5, 4295 (2014).

167. Vopson, M. M. Fundamentals of Multiferroic Materials and Their Possible Applications. Crit. Rev. Solid State Mater. Sci. 40, 223-250 (2015).

168. Meisenheimer, P. B., Novakov, S., Vu, N. M. \& Heron, J. T. Perspective: Magnetoelectric switching in thin film multiferroic heterostructures. J. Appl. Phys. 123, 240901 (2018).

169. Huang, B.-C. et al. Atomically Resolved Electronic States and Correlated Magnetic Order at Termination Engineered Complex Oxide Heterointerfaces. ACS Nano 12, 1089-1095 (2018).

170. Li, T. X. et al. Effect of misfit strain on multiferroic and magnetoelectric properties of epitaxial $\mathrm{La}_{0.7} \mathrm{Sr}_{0.3} \mathrm{MnO}_{3} / \mathrm{BaTiO}_{3}$ bilayer. J. Phys. D. Appl. Phys. 45, 085002 (2012).

171. Hausmann, S. et al. Atomic-scale engineering of ferroelectric-ferromagnetic 
interfaces of epitaxial perovskite films for functional properties. Sci. Rep. 7, 10734 (2017).

172. Guo, H. et al. Interface-induced multiferroism by design in complex oxide superlattices. Proc. Natl. Acad. Sci. 114, 201706814 (2017).

173. Pesquera, D. et al. Surface symmetry-breaking and strain effects on orbital occupancy in transition metal perovskite epitaxial films. Nat. Commun. 3, 1189 (2012).

174. Benckiser, E. et al. Orbital reflectometry of oxide heterostructures. Nat. Mater. 10, 189-193 (2011).

175. Everhardt, A. S., Matzen, S., Domingo, N., Catalan, G. \& Noheda, B. Ferroelectric Domain Structures in Low-Strain $\mathrm{BaTiO}_{3}$. Adv. Electron. Mater. 2, 1500214 (2016).

176. Everhardt, A. S. et al. Temperature-independent giant dielectric response in transitional $\mathrm{BaTiO}_{3}$ thin films. Appl. Phys. Rev. 7, 011402 (2020).

177. Dong, G. et al. Super-elastic ferroelectric single-crystal membrane with continuous electric dipole rotation. Science 366, 475-479 (2019).

178. Nahas, Y. et al. Inverse transition of labyrinthine domain patterns in ferroelectric thin films. Nature 577, 47-51 (2020).

179. Schupper, N. \& Shnerb, N. M. Inverse melting and inverse freezing: A spin model. Phys. Rev. E 72, 046107 (2005).

180. Nadupalli, S., Kreisel, J. \& Granzow, T. Increasing bulk photovoltaic current by strain tuning. Sci. Adv. 5, eaau9199 (2019).

181. Li, D. et al. Superconductivity in an infinite-layer nickelate. Nature 572, 624627 (2019).

182. Catalano, S. et al. Rare-earth nickelates $\mathrm{RNiO}_{3}$ : thin films and heterostructures. Reports Prog. Phys. 81, 046501 (2018).

183. Simons, H. et al. Long-range symmetry breaking in embedded ferroelectrics. Nat. Mater. 17, 814-819 (2018).

184. Xu, X. et al. Variability and origins of grain boundary electric potential detected by electron holography and atom-probe tomography. Nat. Mater. (2020).

185. Mermin, N. D. The topological theory of defects in ordered media. Rev. Mod. Phys. 51, 591-648 (1979).

186. Yadav, A. K. et al. Observation of polar vortices in oxide superlattices. Nature 530, 198-201 (2016).

187. Das, S. et al. Observation of room-temperature polar skyrmions. Nature 568, 368-372 (2019).

188. Erb, K. C. \& Hlinka, J. Vector, Bidirector and Bloch Skyrmion Phases Induced by Structural Crystallographic Symmetry Breaking (2019). arxiv ID: 1910.00075

189. Mühlbauer, S. et al. Skyrmion Lattice in a Chiral Magnet. Science 323, 915919 (2009).

190. Zhao, Z., Ding, X. \& Salje, E. K. H. Flicker vortex structures in multiferroic materials. Appl. Phys. Lett. 105, 112906 (2014).

191. Salje, E. K. H., Li, S., Zhao, Z., Gumbsch, P. \& Ding, X. Polar twin boundaries and nonconventional ferroelectric switching. Appl. Phys. Lett. 106, 212907 (2015).

192. Zykova-Timan, T. \& Salje, E. K. H. Highly mobile vortex structures inside polar twin boundaries in $\mathrm{SrTiO}_{3}$. Appl. Phys. Lett. 104, 082907 (2014).

193. Salje, E. K. H. \& Ishibashi, Y. Mesoscopic structures in ferroelastic crystals: needle twins and right-angled domains. J. Phys. Condens. Matter 8, 8477-8495 
(1996).

194. Pertsev, N. A., Novak, J. \& Salje, E. K. H. Long-range elastic interactions and equilibrium shapes of curved ferroelastic domain walls in crystals. Philos. Mag. A 80, 2201-2213 (2000).

195. Juraschek, D. M. et al. Dynamical Magnetic Field Accompanying the Motion of Ferroelectric Domain Walls. Phys. Rev. Lett. 123, 127601 (2019).

196. Christensen, D. V. et al. Strain-tunable magnetism at oxide domain walls. Nat. Phys. 15, 269-274 (2019).

197. Guo, E.-J., Roth, R., Herklotz, A., Hesse, D. \& Dörr, K. Ferroelectric $180^{\circ}$ Domain Wall Motion Controlled by Biaxial Strain. Adv. Mater. 27, 1615-1618 (2015).

198. McGilly, L. J., Sandu, C. S., Feigl, L., Damjanovic, D. \& Setter, N. Nanoscale Defect Engineering and the Resulting Effects on Domain Wall Dynamics in Ferroelectric Thin Films. Adv. Funct. Mater. 27, 1605196 (2017).

199. Xu, R. et al. Ferroelectric polarization reversal via successive ferroelastic transitions. Nat. Mater. 14, 79-86 (2015).

200. Liu, S., Grinberg, I. \& Rappe, A. M. Intrinsic ferroelectric switching from first principles. Nature 534, 360-363 (2016).

201. Ishibashi, Y. \& Takagi, Y. Note on Ferroelectric Domain Switching. J. Phys. Soc. Japan 31, 506-510 (1971).

202. Ishibashi, Y. \& Orihara, H. A theory of D-E hysteresis loop. Integr. Ferroelectr. 9, 57-61 (1995).

203. Dimmler, K. et al. Switching kinetics in $\mathrm{KNO}_{3}$ ferroelectric thin-film memories. J. Appl. Phys. 61, 5467-5470 (1987).

204. Eliseev, E. A. et al. Screening and retardation effects on $180^{\circ}$-domain wall motion in ferroelectrics: Wall velocity and nonlinear dynamics due to polarization-screening charge interactions. Phys. Rev. B 78, 245409 (2008).

205. Shur, V. Y., Akhmatkhanov, A. R. \& Baturin, I. S. Micro- and nano-domain engineering in lithium niobate. Appl. Phys. Rev. 2, 040604 (2015).

206. Shur, V. Y. Kinetics of ferroelectric domains: Application of general approach to $\mathrm{LiNbO}_{3}$ and $\mathrm{LiTaO}_{3}$. J. Mater. Sci. 41, 199-210 (2006).

207. Bdikin, I. K. et al. Domain dynamics in piezoresponse force spectroscopy: Quantitative deconvolution and hysteresis loop fine structure. Appl. Phys. Lett. 92, 2006-2009 (2008).

208. Rodriguez, B. J. et al. Domain growth kinetics in lithium niobate single crystals studied by piezoresponse force microscopy. Appl. Phys. Lett. 86, 012906 (2005).

209. Gruverman, A., Alexe, M. \& Meier, D. Piezoresponse force microscopy and nanoferroic phenomena. Nat. Commun. 10, 1-9 (2019).

210. Vasudevan, R. K. et al. Domain Wall Conduction and Polarization-Mediated Transport in Ferroelectrics. Adv. Funct. Mater. 23, 2592-2616 (2013).

211. Meier, D., Seidel, J., Gregg, M. \& Ramesh, R. Domain Walls: From Fundamental Properties to Nanotechnology Concepts. (Oxford University Press, 2020).

212. Salje, E. K. H., Xue, D., Ding, X., Dahmen, K. A. \& Scott, J. F. Ferroelectric switching and scale invariant avalanches in $\mathrm{BaTiO}_{3}$. Phys. Rev. Mater. 3, 014415 (2019).

213. Sethna, J. P., Dahmen, K. A. \& Myers, C. R. Crackling noise. Nature 410, 242-250 (2001).

214. Salje, E. K. H. \& Dahmen, K. A. Crackling Noise in Disordered Materials. Annu. Rev. Condens. Matter Phys. 5, 233-254 (2014). 
215. Brezina, B., Fousek, J. \& Glanc, A. Barkhausen pulses in $\mathrm{BaTiO}_{3}$ connected with $90^{\circ}$ switching processes. Czechoslov. J. Phys. 11, 595-601 (1961).

216. Miller, R. C. On the origin of Barkhausen pulses in $\mathrm{BaTiO}_{3}$. J. Phys. Chem. Solids 17, 93-100 (1960).

217. Tan, C. D. et al. Electrical studies of Barkhausen switching noise in ferroelectric PZT: Critical exponents and temperature dependence. Phys. Rev. Mater. 3, 034402 (2019).

218. Puchberger, S. et al. The noise of many needles: Jerky domain wall propagation in $\mathrm{PbZrO}_{3}$ and $\mathrm{LaAlO}_{3}$. APL Mater. 5, 046102 (2017).

219. Soprunyuk, V. et al. Strain intermittency due to avalanches in ferroelastic and porous materials. J. Phys. Condens. Matter 29, 224002 (2017).

220. Harrison, R. J. \& Salje, E. K. H. The noise of the needle: Avalanches of a single progressing needle domain in $\mathrm{LaAlO}_{3}$. Appl. Phys. Lett. 97, 021907 (2010).

221. Casals, B., van Dijken, S., Herranz, G. \& Salje, E. K. H. Electric-field-induced avalanches and glassiness of mobile ferroelastic twin domains in cryogenic $\mathrm{SrTiO}_{3}$. Phys. Rev. Res. 1, 032025 (2019).

222. Casals, B., Nataf, G. F., Pesquera, D. \& Salje, E. K. H. Avalanches from charged domain wall motion in $\mathrm{BaTiO}_{3}$ during ferroelectric switching. $A P L$ Mater. 8, 011105 (2020).

223. Kustov, S., Liubimova, I. \& Salje, E. K. H. Domain Dynamics in QuantumParaelectric $\mathrm{SrTiO}_{3}$. Phys. Rev. Lett. 124, 016801 (2020).

224. Anderson, P. W., Halperin, B. I. \& Varma, C. M. Anomalous low-temperature thermal properties of glasses and spin glasses. Philos. Mag. 25, 1-9 (1972).

225. Kirkpatrick, S. \& Sherrington, D. Infinite-ranged models of spin-glasses. Phys. Rev. B 17, 4384-4403 (1978).

226. Eliseev, E. A. et al. Conductivity of twin-domain-wall/surface junctions in ferroelastics: Interplay of deformation potential, octahedral rotations, improper ferroelectricity, and flexoelectric coupling. Phys. Rev. B 86, 085416 (2012).

227. Eliseev, E. A. et al. Surface effect on domain wall width in ferroelectrics. $J$. Appl. Phys. 106, 084102 (2009).

228. Morozovska, A. N. et al. Thermodynamics of nanodomain formation and breakdown in scanning probe microscopy: Landau-Ginzburg-Devonshire approach. Phys. Rev. B 80, 214110 (2009).

229. Mandel, S. Research suggests a new class of ferroelectric materials. Scilight 2020, 041101 (2020). 